\title{
Transmission of stimulus-locked responses in two coupled phase oscillators
}

\author{
Peter A. Tass* \\ Institute of Medicine, Research Center Jülich, 52425 Jülich, Germany \\ and Department of Stereotaxic and Functional Neurosurgery, University Hospital, 50924 Cologne, Germany
}

(Received 11 August 2003; revised manuscript received 12 December 2003; published 24 May 2004)

\begin{abstract}
A model of two $n: m$ coupled phase oscillators is studied, where both oscillators are subject to random forces, but only one oscillator is repetitively stimulated with a pulsatile stimulus. The focus of the paper is on transmission of transient responses as well as transient synchronization and desynchronization, which are stimulus locked, i.e., tightly time locked to the stimulus. A bistability or multistability of stable synchronized states of the two-phase oscillators (modulo $2 \pi$ ) occurs due to the $n: m$ coupling. Accordingly, after stimulation the two oscillators may tend to qualitatively different stable states, which leads to a cross-trial (CT) response clustering (i.e., a switching between qualitatively different poststimulus responses across trials) of either one of the oscillators or both. A stochastic CT phase resetting analysis allows one to detect such transient responses and provides a reliable estimation of the transmission time. In contrast, CT averaging (averaging over an ensemble of responses), CT standard deviation, and CT cross correlation fail in studying the transmission of such stimulus-locked responses, even in the simpler case of $1: 1$ coupling. In particular, even though being used as golden standard for the analysis of evoked responses in medicine and neuroscience, CT averaging typically causes severe artifacts and misinterpretations.
\end{abstract}

DOI: 10.1103/PhysRevE.69.051909

PACS number(s): 87.19.La, 05.45.Xt, 05.40.Ca

\section{INTRODUCTION}

The impact of stimulation on oscillations and synchronization processes is of great interest in physics [1], chemistry [2], biology [3-5], neuroscience, and medicine [6-9]. The study of transient reactions of neuronal oscillations to pulsatile stimuli is a major approach in neuroscience and a wellestablished tool for clinical diagnosis [6,7]. The golden standard for the univariate analysis of responses of neuronal oscillations to pulsatile stimuli as measured with electroencephalography (EEG), magnetoencephalography (MEG) or local field potentials (LFP) is cross-trial averaging, i.e., averaging over an ensemble of poststimulus responses $[6,10,11]$. The cross-trial $(C T)$ averaged signal of the signal $x_{j}$ of the $j$ th oscillator reads

$$
\bar{x}_{j}(t)=\frac{1}{l} \sum_{k=1}^{l} x_{j}\left(\tau_{k}+t\right),
$$

where the stimulus is repetitively administered at $l$ different onset times $\tau_{1}, \tau_{2}, \ldots, \tau_{l}$. The assumption behind the triggered averaging is that the response $x_{j}$ can be decomposed into a stereotypical evoked response $e_{j}$, which follows the stimulus with a constant delay, plus additive Gaussian noise $\xi_{j}$, so that

$$
x_{j}\left(\tau_{k}+t\right)=e_{j}(t)+\xi_{j}\left(\tau_{k}+t\right)
$$

is fulfilled $[10,11]$. In this case averaging improves the signal-to-noise ratio by $\sqrt{l}$, where the number of responses $l$ typically equals $20-300$, and $\bar{x}_{j}(t) \rightarrow e_{j}(t)$ for $l \rightarrow \infty[10,11]$.

The analysis of evoked responses is still a matter of vivid debate. One issue is the estimation of the time varying fre-

\footnotetext{
*Email address: p.tass@fz-juelich.de
}

quency spectrum of evoked responses. For instance, in the context of self-organized motor control processes [12], similar experiments led to contradictory estimates of conventional power spectra of transient responses [13-15]. It was suggested that these discrepancies might be due to artifacts arising when simple power spectra are calculated for transient responses [14]. In contrast, more sophisticated approaches to spectral estimation and denoising of transient responses are based on autoregressive moving average models [16], on the Wiener filter [17-20], and on the wavelet transform [21-25].

Another fundamental issue of evoked responses is the estimation of their latencies. To study neural information processing and, in particular, the flow of information in interacting neural populations, it is important to analyze the transmission of stimulus-locked responses within networks of interacting neural populations. Along the lines of the averaging approach, transmission is assessed by identifying the timing sequence of stimulus-locked responses of different neuronal populations. This is typically done by determining the difference in time between the occurrence of marker events (such as maxima or minima) of the individual averaged responses belonging to different neuronal populations $[6,10,11]$.

However, the averaging assumption from Eq. (2) is violated by stimulated brain activity for several reasons.

(i) Ongoing oscillations are abundant in the brain [26]. Such oscillators are not "silent" during the prestimulus period.

(ii) Evoked responses, detected with CT averaging, result from reorganizing part of such ongoing oscillations, especially by resetting their phase dynamics $[8,9]$.

(iii) Noise is inevitably inherent in neuronal action and, hence, cannot be modeled appropriately by simply adding it to the deterministic signal [27].

(iv) Coupled phase oscillators, subject to pulsatile stimu- 
lation and noise, may display an antiphase CT response clustering, occurring after a stereotypical reset [28-30]. CT response clustering means that the oscillators switch between qualitatively different responses across trials. In a recent MEG study it has been shown that simple visual stimuli make neuronal populations generate a late antiphase CT response clustering after an early stereotypical reset [31]. The antiphase CT response clustering cannot be detected with CT averaging as defined by Eq. (1), but with a CT analysis based on stochastic phase resetting (see Sec. III).

Accordingly, stimulated neuronal oscillations share basic dynamical features with stimulated phase oscillators (for more details, see Sec. IV A). This paper is dedicated to study transmission of stimulus-locked responses in two coupled phase oscillators, with only one oscillator being stimulated. The motivation behind this approach is to investigate dynamical processes, which are essential for neuronal information processing, in a reasonably simple oscillator model. The insights into the transmission dynamics can then be used for the analysis of experimental data. This approach is intended to make the analysis of evoked response signals less speculative.

In this paper transient dynamical processes will be presented. It will be explained how they can be detected reliably with the CT stochastic phase resetting analysis (Sec. III). In contrast, when applied to study transmission of stimuluslocked responses, the golden standard for such analysis in medicine and neuroscience, the CT averaging from Eq. (1), fails massively. This underlines the importance of using appropriate data analysis techniques as described in Sec. III.

\section{STOCHASTIC MODEL}

\section{A. Two phase oscillators}

I consider a model of two phase oscillators with phases $\psi_{1}, \psi_{2}$ and constant amplitudes obeying

$$
\begin{gathered}
\dot{\psi}_{1}=\omega_{1}-K \sin \left(n \psi_{1}-m \psi_{2}+\theta\right)+X(t) S\left(\psi_{1}\right)+F_{1}(t) \\
\dot{\psi}_{2}=\omega_{2}-K \sin \left(m \psi_{2}-n \psi_{1}-\theta\right)+F_{2}(t)
\end{gathered}
$$

The eigenfrequencies $\omega_{1}$ and $\omega_{2}$ fulfill

$$
n \omega_{1}-m \omega_{2}=\gamma
$$

with detuning $\gamma . K$ is a positive coupling constant, and the coupling is chosen to be symmetrical, i.e., both oscillators are coupled to each other with equal strength. All of the phenomena and mechanisms presented in this paper occur also in the case of nonidentical coupling strength, provided oscillator 2 is coupled to oscillator 1; otherwise the dynamical phenomena studied below cannot be transmitted from oscillator 1 to oscillator 2 . Only oscillator 1 is stimulated, where the stimulus is modeled by a $2 \pi$-periodic, timeindependent function $S\left(\psi_{j}\right)=S\left(\psi_{j}+2 \pi\right)$. In several fields of the natural sciences, especially in biology, the effect of a stimulus is phase dependent $[3,5]$. Switching on and off the stimulus of oscillator 1 is modeled by

$$
X(t)=\left\{\begin{array}{ll}
1 & \text { stimulus is on at time } t \\
0 & \text { stimulus is off at time } t
\end{array} .\right.
$$

The random forces $F_{1}$ and $F_{2}$ are Gaussian white noise fulfilling $\left\langle F_{j}(t)\right\rangle=0$ and $\left\langle F_{j}(t) F_{k}(\widetilde{t})\right\rangle=D \delta_{j k} \delta(t-\widetilde{t})$ with constant noise amplitude $D$. Equations (3) and (4) may serve as a minimal model for two neurons [5], one of them being electrically stimulated, or as a minimal model for two interacting neuronal populations, where only one of them is directly affected by a sensory stimulus as discussed below. The amplitude of both oscillators is set equal to 1 , so that the signal of the $j$ th phase oscillator reads

$$
x_{j}(t)=\cos \psi_{j}(t) .
$$

\section{B. Spontaneous dynamics}

Let me dwell on the oscillators' synchronization behavior occurring spontaneously, i.e., without stimulation. For this I set $X=0$ in Eq. (3). The evolution equation of the $n: m$ phase difference

$$
\Phi_{n, m}=n \psi_{1}-m \psi_{2}
$$

reads

$$
\dot{\Phi}_{n, m}=\gamma-(n+m) K \sin \left(\Phi_{n, m}+\theta\right)+F(t),
$$

where $\gamma$ is the detuning from Eq. (5). The random force $F(t)=n F_{1}(t)-m F_{2}(t)$ is Gaussian white noise fulfilling $\langle F(t)\rangle=0$ and $\langle F(t) F(\widetilde{t})\rangle=\left(n^{2}+m^{2}\right) D \delta(t-\widetilde{t})$.

In the noise-free case $(D=0)$ the dynamics is governed by a potential

$$
V\left(\Phi_{n, m}\right)=-\int_{c}^{\Phi_{n, m}} G(\xi) d \xi,
$$

where $G\left(\Phi_{n, m}\right)=\gamma-(n+m) K \sin \left(\Phi_{n, m}+\theta\right)$, and $c$ is a constant, so that

$$
\dot{\Phi}_{n, m}=-\frac{d V\left(\Phi_{n, m}\right)}{d \Phi_{n, m}} .
$$

With suitably chosen $c$ one gets

$$
V\left(\Phi_{n, m}\right)=-\gamma \Phi_{n, m}-(n+m) K \cos \left(\Phi_{n, m}+\theta\right) .
$$

For $\left|n \omega_{1}-m \omega_{2}\right|<(n+m) K$ the potential $V$ has a local maximum and a local minimum, which correspond to an unstable fixed point of $\Phi_{n, m}$ and a stable fixed point of $\Phi_{n, m}$, denoted by $\Phi_{n, m}^{\mathrm{u}}$ and $\Phi_{n, m}^{\mathrm{s}}$, respectively. Maximum and minimum are determined by setting $d V / d \Phi_{n, m}=0$, which with Eq. (5) yields

$$
\Phi_{n, m}^{\mathrm{u}, \mathrm{s}}=\arcsin \frac{n \omega_{1}-m \omega_{2}}{(n+m) K}-\theta,
$$

where one of the two solutions of the arcsin belongs to the unstable fixed point and the other one to the stable fixed point. The dynamics given by Eq. (11) corresponds to an 
overdamped motion of a particle in the potential $V$. $\Phi_{n, m}$ moves in such a way that it minimizes $V\left(\Phi_{n, m}\right)$, and $\Phi_{n, m}$ stops only when $d V / d \Phi_{n, m}$ vanishes (see Ref. [1] and Chap. 9 in Ref. [32]).

With respect to the stable synchronized state, there is an important difference between $1: 1$ coupling and $n: m$ coupling (with $n \neq 1$ and/or $m \neq 1$ and $n \neq m$ ): In case of the $1: 1$ coupling $\Phi_{1,1}^{\mathrm{s}}$ belongs to one stable solution of the phases $\psi_{1}$ and $\psi_{2}$ (modulo $2 \pi$ ). In contrast, with $n: m$ coupling $\Phi_{n, m}^{\mathrm{s}}$ corresponds to more than only one stable solution of the phases $\psi_{1}$ and $\psi_{2}$ (modulo $2 \pi$ ). For instance, with $n=1$ and $m=2$, for given $\psi_{1}$ the two stable solutions for $\psi_{2}$ read $\psi_{2}$ $=\left(\psi_{1}-\Phi_{n, m}^{\mathrm{s}}\right) / 2$ and $\psi_{2}=\left(\psi_{1}-\Phi_{n, m}^{\mathrm{s}}\right) / 2+\pi$. Analogously, in case of $n=1$ and $m=3$, for given $\psi_{1}$ the three stable solutions of $\psi_{2}$ read $\psi_{2}=\left(\psi_{1}-\Phi_{n, m}^{\mathrm{s}}\right) / 3, \psi_{2}=\left(\psi_{1}-\Phi_{n, m}^{\mathrm{s}}\right) / 3+2 \pi / 3$, and $\psi_{2}=\left(\psi_{1}-\Phi_{n, m}^{\mathrm{s}}\right) / 3+4 \pi / 3$. This bistability and multistability is important for understanding the poststimulus responses studied below (Secs. VII-IX).

Adding noise to the system $(D>0)$ changes the situation completely. The trajectory of the particle can no longer be predicted. Rather the particle's dynamics has to be described in a probabilistic sense. For instance, with the Fokker-Planck equation belonging to Eq. (9) the time course of the probability density $p\left(\Phi_{n, m}, t\right)$ can be determined. $p\left(\Phi_{n, m}, t\right) d \Phi_{n, m}$ provides the probability of finding $\Phi_{n, m}$ in the interval $\left[\Phi_{n, m}, \Phi_{n, m}+d \Phi_{n, m}\right]$ (see Ref. [1] and Chap. 9 in Ref. [32]). For an analysis of the influence of pulsatile stimulation $[X$ $=1$ in Eq. (3)] on the noisy dynamics of $\Phi_{n, m}$ in the doublewell potential $V$ from Eq. (12), I refer to Refs. [29,30].

\section{CROSS-TRIAL ANALYSIS BASED ON STOCHASTIC PHASE RESETTING}

\section{A. Cross-trial analysis}

I introduce normalized phases

$$
\phi_{j}(t)=\frac{\psi_{j}(t)}{2 \pi} \bmod 1 \quad(j=1,2)
$$

and the normalized cyclic $n: m$ phase difference

$$
\varphi_{n, m}(t)=\frac{n \psi_{1}(t)-m \psi_{2}(t)}{2 \pi} \bmod 1 .
$$

I want to detect whether in an ensemble of responses to the stimulus there are epochs during which the phases $\phi_{1}, \phi_{2}$ and/or the phase difference $\varphi_{n, m}$ display a stereotypical, tightly stimulus-locked time course. To this end, I deliver a series of $l$ identical stimuli to oscillator 1 at random times $\tau_{1}, \tau_{2}, \ldots, \tau_{l}$ as shown in Fig. 1. The length of the interstimulus intervals (ISI) is randomized according to

$$
\tau_{k+1}-\tau_{k}=t_{\operatorname{win}}+\zeta_{k},
$$

where the minimal ISI, $t_{\mathrm{win}}$, is constant and large compared to the stimulation duration as well as the time scale of the transient dynamics. $\zeta_{k}$ is uniformly distributed in $[0, k 2 \pi / \omega]$, where $k$ is a small integer. I attach an identical time window $\left[t_{a}, t_{b}\right]$ to each stimulus $\left(t_{a}<0, t_{b}>0\right.$, Fig. 1$)$. Each window has a time axis $t^{\prime}$, where $t^{\prime} \in\left[t_{a}, t_{b}\right]$. The onset of the stimu-

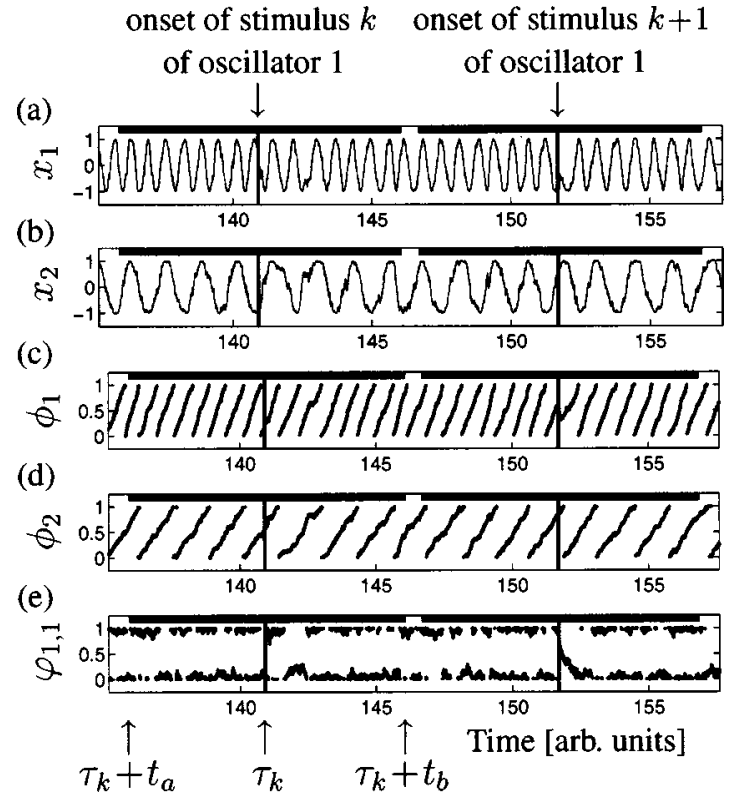

FIG. 1. The cross-trial analysis is illustrated schematically. A series of $l$ identical stimuli is administered to oscillator 1 at random times $\tau_{1}, \tau_{2}, \ldots, \tau_{l}$. Onsets of the stimuli of oscillator 1 are indicated by solid vertical lines. An identical time window $\left[t_{a}, t_{b}\right]$ (with $t_{a}<0$, $t_{b}>0$ ) is attached to each stimulus and indicated by a shaded region at the top of each panel. The signals $x_{1}(t)=\cos \left[2 \pi \phi_{1}(t)\right]$ and $x_{2}(t)$ $=\cos \left[2 \pi \phi_{2}(t)\right]$ defined by Eq. (7) are displayed in (a) and (b). The phases $\phi_{1}$ and $\phi_{2}$ from Eq. (14) are shown in (c) and (d). The normalized cyclic $1: 1$ phase difference $\varphi_{1,1}$ from Eq. (15) is shown in (e). Continuous variations around $\varphi_{1,1}=0$ appear as abrupt jumps between 0 and 1 because $\varphi_{1,1}=0$ and $\varphi_{1,1}=1$ are identical. The traces shown are obtained by numerical integration of the model given by Eqs. (3) and (4) with parameters as in Fig. 5. In this paper Eqs. (3) and (4) are numerically solved with Euler's technique and a time step of 0.0005 .

lus in each window lies in $t^{\prime}=0$. The window length $t_{b}-t_{a}$ is smaller than the length of the ISI $\left(t_{b}-t_{a}<t_{\text {win }}\right)$, but large compared to the time scale of the transient dynamics.

For the sake of simplicity I drop the prime in $t^{\prime}$, so that from now on $t$ denotes the time axis of the window. To evaluate the dynamics of the ensemble of stimulus-locked responses statistically, I collect the values for $\phi_{j}$ and $\varphi_{n, m}$ across all trials for each time $t$ relative to stimulus onset. Accordingly, for each time $t \in\left[t_{a}, t_{b}\right] \mathrm{I}$ introduce the timedependent $C T$ distributions of the normalized phases from Eq. (14) and the cyclic $n: m$ phase difference from Eq. (15) by

$$
\left\{\phi_{j}\left(t+\tau_{k}\right)\right\}_{k=1, \ldots, l}, \quad\left\{\varphi_{n, m}\left(t+\tau_{k}\right)\right\}_{k=1, \ldots, l} .
$$

The time course of $\phi_{j}$ and $\varphi_{n, m}$ is perfectly stimulus locked at time $t$ if the corresponding CT distributions from Eq. (17) are Dirac-like distributions, i.e., $\phi_{j}\left(t+\tau_{i}\right)=\phi_{j}\left(t+\tau_{k}\right)$ and $\varphi_{n, m}\left(t+\tau_{i}\right)=\varphi_{n, m}\left(t+\tau_{k}\right)$ for all $i, k=1, \ldots, l$. If $\phi_{j}$ and $\varphi_{n, m}$ are not at all stimulus locked at time $t$, these distributions are uniform. 


\section{B. Stimulus-locking indices}

The extent of stimulus locking of $\phi_{j}$ and $\varphi_{n, m}$ is quantified for each time $t$ by means of the time-dependent stimuluslocking indices $\lambda_{j}^{(\nu)}(t)$ of $\phi_{j}$ given by

$$
\lambda_{j}^{(\nu)}(t)=\left|\frac{1}{l} \sum_{k=1}^{l} \exp \left[i \nu 2 \pi \phi_{j}\left(\tau_{k}+t\right)\right]\right|
$$

and the $n: m$ synchronization index $\sigma_{n, m}(t)$ of $\varphi_{n, m}$ given by

$$
\sigma_{n, m}(t)=\left|\frac{1}{l} \sum_{k=1}^{l} \exp \left[i 2 \pi \varphi_{n, m}\left(\tau_{k}+t\right)\right]\right|,
$$

where $|y|$ denotes the modulus of $y$, and $\nu$ is an integer [28-30]. $\lambda_{j}^{(\nu)}(t)$ detects whether $\phi_{j}$ 's CT distribution from Eq. (17) at time $t$ has $\nu$ peaks that are equally spaced in $[0,1]$ (modulo 1). With $\sigma_{n, m}(t)$ I detect whether $\varphi_{n, m}$ 's CT distribution from Eq. (17) at time $t$ has one prominent peak. 0 $\leqslant \lambda_{j}^{(\nu)}(t) \leqslant 1,0 \leqslant \sigma_{n, m}(t) \leqslant 1$ are fulfilled for $t \in\left[t_{a}, t_{b}\right]$ and for all integer $\nu . \lambda_{j}^{(\nu)}$ and $\sigma_{n, m}$ are the modulus of the $\nu$ th and the first Fourier mode of the corresponding CT distributions from Eq. (17), respectively (see Ref. [5]).

I consider the three leading indices $(\nu=1,2,3)$ in different characteristic situations.

(i) If the distribution $\left\{\phi_{j}\left(t+\tau_{k}\right)\right\}_{k=1, \ldots, l}$ at time $t$ is uniform, $\lambda_{j}^{(\nu)}(t)=0$ for $\nu=1,2,3$.

(ii) One pronounced peak of the distribution $\left\{\phi_{j}(t\right.$ $\left.\left.+\tau_{k}\right)\right\}_{k=1, \ldots, l}$ at time $t$ corresponds to large $\lambda_{j}^{(\nu)}(t)$ for $\nu$ $=1,2,3$.

(iii) Two pronounced antiphase peaks of the distribution $\left\{\phi_{j}\left(t+\tau_{k}\right)\right\}_{k=1, \ldots, l}$ at time $t$ are characterized by large $\lambda_{l}^{(2)}(t)$ and small $\lambda_{j}^{(\nu)}(t)$ for $\nu=1,3$.

(iv) Three pronounced and equally spaced peaks of the distribution $\left\{\phi_{j}\left(t+\tau_{k}\right)\right\}_{k=1, \ldots, l}$ at time $t$ are connected with a large $\lambda_{j}^{(3)}(t)$ and small $\lambda_{j}^{(\nu)}(t)$ for $\nu=1,2$.

Based on Eq. (18) I introduce indices which detect specific configurations of the CT distribution of the phase: A resetting stimulus puts an oscillator to a particular phase. Therefore the index $\lambda_{j}^{(1)}$ serves as a resetting index of the $j$ th oscillator, denoted by

$$
\rho_{j}(t)=\lambda_{j}^{(1)}(t)
$$

Two symmetric antiphase peaks of the distribution $\left\{\phi_{j}(t\right.$ $\left.\left.+\tau_{k}\right)\right\}_{k=1, \ldots, l}$ at time $t$ are specifically detected with the timedependent antiphase CT clustering index of the jth oscillator defined by

$$
\alpha_{j}(t)=\lambda_{j}^{(2)}(t)-\lambda_{j}^{(1)}(t),
$$

Ref. [30]. $-1 \leqslant \alpha_{j}(t) \leqslant 1$ is fulfilled for all times $t$, where two Dirac-like symmetric antiphase peaks are related to $\alpha_{j}(t)=1$. Analogously, three equally spaced symmetric peaks of the distribution $\left\{\phi_{j}\left(t+\tau_{k}\right)\right\}_{k=1, \ldots, l}$ at time $t$ are detected by means of the time-dependent $C T$ three-clustering index of the jth oscillator defined by

$$
\beta_{j}(t)=\lambda_{j}^{(3)}(t)-\lambda_{j}^{(1)}(t)
$$

$-1 \leqslant \beta_{j}(t) \leqslant 1$ is fulfilled for all times $t$, where three Diraclike symmetric and equally spaced peaks are connected with $\beta_{j}(t)=1$.

Geometrically speaking, the time-dependent stimulus locking indices $\lambda_{j}^{(\nu)}(t)$ of $\phi_{j}$ from Eq. (18) represent a timedependent distance between the origin of the Gaussian plane and the center of mass of the CT distribution $\left\{\exp \left[i \nu 2 \pi \phi_{j}\left(\tau_{k}+t\right)\right]\right\}_{k=1, \ldots, l}$. Inspired by circular statistics [33], additionally, one can estimate the dispersion of the CT distribution $\left\{\exp \left[i \nu 2 \pi \phi_{j}\left(\tau_{k}+t\right)\right]\right\}_{k=1, \ldots, l}$ in a complementary way. For this, I use the formulas for the mean angular deviation of unimodal and antiphase bimodal distributions [34]. This enables one to introduce the CT mean angular deviation $\Lambda_{j}^{(1)}(t)$ for a unimodal CT distribution $\left\{\phi_{j}\left(t+\tau_{k}\right)\right\}_{k=1, \ldots, l}$ at time $t$ and the CT mean angular deviation $\Lambda_{j}^{(2)}(t)$ for an antiphase bimodal CT distribution $\left\{\phi_{j}\left(t+\tau_{k}\right)\right\}_{k=1, \ldots, l}$ at time $t$ defined by

$$
\Lambda_{j}^{(\nu)}(t)=f_{\nu} \sqrt{2\left[1-\lambda_{j}^{(\nu)}(t)\right]},
$$

where $f_{1}=1$ and $f_{2}=0.5$. The mean angular deviation is equivalent to the standard deviation in linear statistics $[33,34]$. Analogously, one can introduce the CT mean angular deviation $\Upsilon_{n, m}(t)$ for a unimodal CT distribution $\left\{\varphi_{n, m}(t\right.$ $\left.\left.+\tau_{k}\right)\right\}_{k=1, \ldots, l}$ at time $t$ by setting

$$
Y_{n, m}(t)=\sqrt{2\left[1-\sigma_{n, m}(t)\right]} .
$$

By definition the mean angular deviations from Eqs. (23) and (24) are complementary to the corresponding stimulus locking indices from Eqs. (18) and (19). This will be demonstrated in Sec. VII B.

Apart from the indices defined by Eqs. (18)-(22), I use indices based on the Shannon entropy $[5,30]$ in order to quantify the deviation of the distributions $\left\{\phi_{j}\left(t+\tau_{k}\right)\right\}_{k=1, \ldots, l}$ and $\left\{\varphi_{n, m}\left(t+\tau_{k}\right)\right\}_{k=1, \ldots, l}$ from a uniform one in a more general way. The time-dependent entropy based stimulus locking in$\operatorname{dex} \mu_{j}(t)$ of $\phi_{j}$ reads

$$
\mu_{j}(t)=\frac{S_{\max }-S_{j}(t)}{S_{\max }},
$$

where $S_{j}(t)=-\sum_{i=1}^{N} p_{i} \ln p_{i}$ is the entropy of the distribution $\left\{\phi_{j}\left(t+\tau_{k}\right)\right\}_{k=1, \ldots, l}$ at time $t$, and $p_{i}$ denotes the relative frequency of finding $\phi_{j}\left(t+\tau_{k}\right)$ within the $i$ th bin. $S_{\max }=\ln N$ is the entropy of a uniform distribution, where $N=\exp [0.626$ $+0.4 \ln (l-1)]$ is the optimal number of bins [35], and $l$ is the number of stimuli administered. $0 \leqslant \mu_{j}(t) \leqslant 1$ holds for all $t$, where $\mu_{j}(t)=0$ corresponds to a uniform distribution (no stimulus locking) at time $t$, whereas $\mu_{j}(t)=1$ corresponds to a Dirac-like distribution (perfect stimulus locking) at time $t$.

The time-dependent entropy based $n: m$ synchronization index $\eta_{n, m}(t)$ of $\varphi_{n, m}$ is given by 


$$
\eta_{n, m}(t)=\frac{S_{\max }-S_{n, m}(t)}{S_{\max }},
$$

where $S_{n, m}(t)$ is the entropy of the distribution $\left\{\varphi_{n, m}(t\right.$ $\left.\left.+\tau_{k}\right)\right\}_{k=1, \ldots, l}$ at time $t$.

The first and the 99th percentile of the prestimulus distributions of the indices $\left\{\rho_{j}(t)\right\}_{t \in\left[t_{a}, 0[\right.}, \quad\left\{\alpha_{j}(t)\right\}_{t \in\left[t_{a}, 0[\right.}$, $\left\{\beta_{j}(t)\right\}_{t \in\left[t_{a}, 0[\right.}, \quad\left\{\sigma_{n, m}(t)\right\}_{t \in\left[t_{a}, 0[\right.}, \quad\left\{\mu_{j}(t)\right\}_{t \in\left[t_{a}, 0[\right.}, \quad\left\{\eta_{n, m}(t)\right\}_{t \in\left[t_{a}, 0[\right.}$, $\left\{\Lambda_{j}^{(\nu)}(t)\right\}_{t \in\left[t_{a}, 0[\right.}$, and $\left\{Y_{n, m}(t)\right\}_{t \in\left[t_{a}, 0[\right.}$ serve as confidence levels in order to determine whether a stimulus causes a significant increase or decrease of the corresponding locking index. For example, a pronounced reset, i.e., an increase of the stimulus locking of $\phi_{j}$ at time $t$, is considered significant provided $\rho_{j}(t)$ is greater than the 99th percentile of the prestimulus

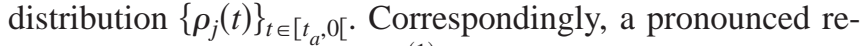
set leads to a decrease of $\Lambda_{j}^{(1)}$ below its first percentile (see Sec. VII B). Significant stimulus-locked synchronization or desynchronization at time $t$ occurs provided $\sigma_{n, m}(t)$ exceeds the 99th percentile or falls below the first percentile of $\left\{\sigma_{n, m}(t)\right\}_{t \in\left[t_{a}, 0[\text {. The differences between the above men- }\right.}$ tioned indices will be discussed below.

\section{Tests for randomness}

In addition to the stimulus locking indices described in Sec. II B, for the evaluation of the CT distributions from Eq. (17) I use a statistical approach, which has been developed in the field of circular statistics [31]. The starting point of this approach is an observed distribution of $l$ phases, which will be denoted by

$$
\left\{\Psi_{1}, \Psi_{2}, \ldots, \Psi_{l}\right\},
$$

where the phase $\Psi_{j}$ is unwrapped and not normalized (i.e., not divided by $2 \pi$ ) for $j=1, \ldots, l$. The corresponding circular distribution reads

$$
\left\{\exp \left(i \Psi_{1}\right), \exp \left(i \Psi_{2}\right), \ldots, \exp \left(i \Psi_{l}\right)\right\} .
$$

From the geometrical point of view, the distribution given by Eq. (28) can be considered as a distribution of unit vectors in the complex plane. To determine whether this distribution has a preferred orientation, one determines the mean vector

$$
R \exp (i \Theta)=\frac{1}{l} \sum_{k=1}^{l} \exp \left(i \Psi_{k}\right),
$$

where $R$ is the length and $\Theta$ is the orientation of the mean vector. For the sake of illustration, one may assume that each unit vector is related to a mass $M$ located in $\exp \left(i \Psi_{j}\right)(j$ $=1, \ldots, l)$. In this case the center of mass is located in $R \exp (i \Theta)$ in the complex plane.

A basic task in circular statistics is to study the directedness of the circular distribution from Eq. (28), i.e., to answer the following question: Is the observed circular distribution significantly different from randomness. More precisely, is there statistical evidence of one sidedness or directedness? Put otherwise, is the location of the center of mass significantly different from the origin of the Gaussian plane $(R>0)$ ?
Several statistical tests have been designed which enable one to test whether there is statistical evidence of directedness in unimodal distributions. Examples of this kind of statistical tests are the Rayleigh test (see Ref. [33]), the Hodges and Ajne's test [36-38], Rao's spacing test [39], the range test [40], the $\chi^{2}$ test [41], Watson's $U_{n}^{2}$ test [42], and the Kuiper test [43-46].

The null hypothesis of such tests is that there is randomness, i.e., the observed distribution is uniform. Applying such a test yields the $P$ value, which is the smallest significance level by which the null hypothesis can be rejected. Accordingly, if the observed distribution is a uniform distribution, one obtains $P=1$. In contrast, if the observed distribution has one pronounced peak, one gets a $P$ value close to 0 .

These tests work well for unimodal distributions, i.e., for distributions with one peak. In the case of multimodal distributions (distributions with more than one peak) the situation is different.

(i) If a multimodal distribution has $\nu$ equally spaced peaks, for example, two antiphase peaks $(\nu=2)$, one removes the mulitmodality by the replacement

$$
\Psi_{j} \rightarrow \nu \Psi_{j}
$$

Instead of Eqs. (27) and (28) the distribution of the phases and the corresponding circular distribution then read $\left\{\nu \Psi_{1}, \nu \Psi_{2}, \ldots, \nu \Psi_{l}\right\} \quad$ and $\quad\left\{\exp \left(i \nu \Psi_{1}\right), \exp \left(i \nu \Psi_{2}\right), \ldots\right.$, $\left.\exp \left(i \nu \Psi_{l}\right)\right\}$. The mean vector of such a scaled distribution is

$$
R_{\nu} \exp \left(i \Theta_{\nu}\right)=\frac{1}{l} \sum_{k=1}^{l} \exp \left(i \nu \Psi_{k}\right)
$$

For $\nu=1$ (i.e., in the case of only one peak) Eqs. (29) and (31) coincide: $R=R_{1}, \Theta=\Theta_{1}$. Due to the replacement from Eq. (30) a multimodal distribution $\left\{\Psi_{1}, \Psi_{2}, \ldots, \Psi_{l}\right\}$ with $\nu$ equally spaced peaks turns into a unimodal distribution $\left\{\nu \Psi_{1}, \nu \Psi_{2}, \ldots, \nu \Psi_{l}\right\}$. In this way, the replacement from Eq. (30) makes it possible to apply all above mentioned tests for randomness to the unimodal distribution $\left\{\nu \Psi_{1}, \nu \Psi_{2}, \ldots, \nu \Psi_{l}\right\}$.

Note, this trick, i.e., transforming an equally spaced multimodal distribution to a unimodal distribution by means of Eq. (30), is exactly the same reasoning that has been used for the detection of $\nu$ equally spaced peaks in a CT distribution by means of the stimulus locking indices from Eqs. (18) and (19): With $\Psi_{k} \rightarrow 2 \pi \phi_{j}\left(\tau_{k}+t\right)$ one immediately gets $R_{\nu}=\lambda_{j}^{(\nu)}$ $\times(t)$.

(ii) If a multimodal distribution has $\nu$, not necessarily equally spaced peaks, the replacement from Eq. (30) will not lead to a unimodal distribution. In this general case several of the above mentioned tests will not fly. However, we can still use Rao's spacing test [39], the $\chi^{2}$ test [41], Watson's $U_{n}^{2}$ test [42], and the Kuiper test [43-46]. All of these tests detect whether the observed distribution differs significantly from randomness. For this, the null hypothesis is that the observed distribution is uniform. 
This statistical approach corresponds to estimating how strongly the observed CT distribution differs from a uniform CT distribution by means of the entropy based stimulus locking indices from Eqs. (25) and (26). The entropy based method compares the observed distribution with the uniform distribution irrespective of the number of peaks of the observed distribution (see Sec. III B).

Statistical tests of this kind found numerous applications to physics and biology [33]. For instance, in physiology statistical tests have been used to detect cardiorespiratory synchronization [47-49]. In this case $\Psi_{1}, \Psi_{2}, \ldots, \Psi_{l}$ denote the phase difference between the cardiac and the respiratory signals measured at consecutive times $t_{1}, t_{2}, \ldots, t_{l}$ during an observation. Also for the study of movement coordination statistical tests have been applied [50].

\section{Cross-trial Kolmogorov-Smirnov test}

The most accepted statistical test for continuous data is the Kolmogorov-Smirnov test [51]. In this study the Kolmogorov-Smirnov test is used in a cross-trial manner. To test whether the observed CT distribution of the phase of oscillator $j,\left\{\phi_{j}\left(t+\tau_{k}\right)\right\}_{k=1, \ldots, l}$ from Eq. (17), differs significantly from randomness, i.e., from a uniform distribution, I determine the corresponding $P$ value denoted by $b_{j}$. Analogously, to test whether the observed CT distribution of the $n: m$ phase difference, $\left\{\varphi_{n, m}\left(t+\tau_{k}\right)\right\}_{k=1, \ldots, l}$ from Eq. (17), differs significantly from a uniform distribution, the corresponding $P$ value $B_{n, m}$ is determined.

I explain the application of the Kolmogorov-Smirnov test to the CT distribution of the phase of oscillator $j$. It works similarly for the CT distribution of the $n: m$ phase difference. For each potential value $\phi$, the CT Kolmogorov-Smirnov test compares the proportion of values $\phi_{j}\left(t+\tau_{1}\right), \phi_{j}(t$ $\left.+\tau_{2}\right), \ldots, \phi_{j}\left(t+\tau_{l}\right)$, which are less than $\phi$, with the corresponding proportion of phase values of a uniform distribution less than $\phi . G(\phi)$ is the cumulative distribution function of the observed probability distribution. Put otherwise, $G(\phi)$ is the function giving the fraction of the data points $\phi_{j}(t$ $\left.+\tau_{1}\right), \phi_{j}\left(t+\tau_{2}\right), \ldots, \phi_{j}\left(t+\tau_{l}\right)$, which is left of $\phi . P(\phi)$ is the cumulative distribution function of a uniform distribution.

The Kolmogorov-Smirnov test uses the maximal value $D$ of the absolute difference between the two cumulative distribution functions

$$
D=\max _{0 \leqslant \phi \leqslant 1}|G(\phi)-P(\phi)| .
$$

The probability of error (i.e., the probability value) for rejecting the null hypothesis then reads

$$
P_{K S}(d)=2 \sum_{j=1}^{\infty}(-1)^{j-1} \exp \left(-2 j^{2} d^{2}\right)
$$

where

$$
d=D\left(\sqrt{\frac{l}{2}}+0.12+0.11 \sqrt{\frac{2}{l}}\right) .
$$

The $P$ values of the CT Kolomogorov-Smirnov tests and their corresponding CT distributions from Eq. (17) are denoted by

$$
\begin{gathered}
b_{j}(t) \text { for } \quad\left\{\phi_{j}\left(t+\tau_{k}\right)\right\}_{k=1, \ldots, l}, \\
B_{n, m}(t) \text { for } \quad\left\{\varphi_{n, m}\left(t+\tau_{k}\right)\right\}_{k=1, \ldots, l} .
\end{gathered}
$$

The $P$ value provides the smallest significance level by which the null hypothesis (i.e., randomness) can be rejected. Hence, one gets $P=1$, provided the observed distribution is uniform. If the observed CT distribution has one Dirac-like peak, one obtains a $P$ value close to 0 . Multimodal CT distributions yield values of $P$ somewhere in between 0 and 1 (see below). Note, from the $P$ value one cannot infer the number of peaks of a multimodal distribution.

In Secs. VII E and X the CT Kolomogorov-Smirnov test will be compared to the stimulus locking indices from Sec. III B and to the CT Kuiper test described below.

\section{E. Cross-trial Kuiper test}

The Kuiper test is a circular version of the KolmogorovSmirnov test [33,43-46]. To account for the circular nature of phases or phase differences, the Kuiper test does not use the maximal value $D$ of the absolute difference between the two cumulative distribution functions [see Eq. (32)]. Instead, the Kuiper test uses $V=D^{+}+D^{-}$, where

$$
\begin{aligned}
& D^{+}=\max _{0 \leqslant \phi \leqslant 1} G(\phi)-P(\phi), \\
& D^{-}=\max _{0 \leqslant \phi \leqslant 1} P(\phi)-G(\phi) .
\end{aligned}
$$

For further details I refer to Refs. [33,43-46]. The $P$ values of the CT Kuiper tests and their corresponding CT distributions from Eq. (17) read

$$
\begin{gathered}
p_{j}(t) \text { for } \quad\left\{\phi_{j}\left(t+\tau_{k}\right)\right\}_{k=1, \ldots, l}, \\
P_{n, m}(t) \text { for }\left\{\varphi_{n, m}\left(t+\tau_{k}\right)\right\}_{k=1, \ldots, l} .
\end{gathered}
$$

In Secs. V B and VII E the CT Kuiper test will be compared to the stimulus-locking indices from Sec. III B. In Secs. VII E and X it will be explained why it is absolutely necessary to use the CT Kuiper test instead of the CT Kolmogorov Smirnov test.

The first and the 99th percentile of the prestimulus distributions $\left\{p_{j}(t)\right\}_{t \in\left[t_{a}, 0[\right.}$ and $\left\{P_{n, m}(t)\right\}_{t \in\left[t_{a}, 0[\right.}$ provide confidence levels, which allow one to determine whether a stimulus causes a significant change of the corresponding CT distribution of the phase or the phase difference.

\section{STANDARD ANALYSIS APPLIED ACROSS TRIALS}

In this section I sketch how standard univariate and bivariate data analysis are applied across trials.

\section{A. Cross-trial averaging}

$\mathrm{CT}$ averaging relative to stimulus onset is widely used for noise reduction of biological signals, such as EEG $[7,10]$ and 
MEG signals [11,26] as well as LFPs [6]. In fact, CT averaging is the golden standard in neuroscience and medicine for the detection of stimulus-locked responses of an oscillator $[7,10,11]$. The CT averaged signal of the $j$ th phase oscillator is defined by Eq. (1) and reads $\bar{x}_{j}(t)=l^{-1} \sum_{k=1}^{l} x_{j}\left(\tau_{k}+t\right)$ where the signal of the $j$ th oscillator is given by Eq. (7). The stimulus onsets $\tau_{1}, \tau_{2}, \ldots, \tau_{l}$ serve as trigger points for the averaging procedure.

As discussed in Sec. I, calculating the CT averaged signal is only justified, provided the response $x_{j}$ under consideration can be decomposed into a stereotypical evoked response $e_{j}$, which follows the stimulus with a constant delay, and additive Gaussian noise $\xi_{j}$ according to Eq. (2) $[10,11]$.

The stochastic oscillator model given by Eqs. (3) and (4) violates the averaging assumption given by Eq. (2).

(i) The oscillators perform an ongoing oscillation, so that it is not appropriate to assume that their signal $x_{j}$ is a deterministic response only during a short period of time, and nothing but noise during the rest of the time.

(ii) The stimulation effect may depend on the phase of the oscillator.

(iii) Noise is not simply added to the signal $x_{j}$, but inherent in the dynamics.

(iv) The oscillators may display a CT response clustering instead of reacting just stereotypically. Anyhow, all these features, which are in contradiction to the averaging assumption from Eq. (2), are basic dynamical features which both the model given by Eqs. (3) and (4) and oscillatory neuronal activity have in common (see discussion in Sec. I). In a nutshell, the averaging assumption given by Eq. (2) is not fulfilled for the phase oscillator model under consideration as well as for stimulated neuronal oscillations. And, the phase oscillator model may serve as a simple model for stimulated oscillatory brain activity.

\section{B. Cross-trial standard deviation}

To estimate whether the poststimulus signals of the oscillators are stereotypical, I determine the standard deviation across trials at each time $t$ relative to stimulus onset with the cross-trial standard deviation of the $j$ th oscillator according to

$$
\varrho_{j}(t)=\sqrt{\frac{1}{l-1} \sum_{k=1}^{l}\left[x_{j}\left(\tau_{k}+t\right)-\bar{x}_{j}(t)\right]^{2}},
$$

where $\bar{x}_{j}$ is the CT averaged signal from Eq. (1), and $x_{j}(t)$ $=\cos \left[2 \pi \phi_{j}(t)\right]$ according to Eqs. (7) and (14). $\varrho_{j}(t)$ is expected to be small when the signals $x_{j}\left(\tau_{k}+t\right)$ are perfectly stimulus locked at time $t$, and large when there is no stimulus locking.

\section{Cross-trial cross correlation}

To reveal stimulus-locked linear correlations between the two oscillators I determine the cross correlation across trials at each time $t$ relative to stimulus onset with the cross-trial cross-correlation (CTCC) between $x_{1}$ and $x_{2}$ at time $t$ given by

$$
C(t)=\frac{\sum_{k=1}^{l} x_{1}\left(t+\tau_{k}\right) x_{2}\left(t+\tau_{k}\right)}{\sqrt{\left[\sum_{k=1}^{l} x_{1}^{2}\left(t+\tau_{k}\right)\right]\left[\sum_{k=1}^{l} x_{2}^{2}\left(t+\tau_{k}\right)\right]}} .
$$

By definition $C$ is set to zero if all responses of $x_{1}$ or $x_{2}$ vanish at time $t$, in order to avoid a singularity. To avoid singular behavior, alternatively, one can use the cross-trial sign cross correlation (CTSCC) between $x_{1}$ and $x_{2}$ at time $t$ defined by

$$
S(t)=\frac{1}{l} \sum_{k=1}^{l} \operatorname{sgn}\left[x_{1}\left(t+\tau_{k}\right) x_{2}\left(t+\tau_{k}\right)\right],
$$

which corresponds to the CTCC of the signals' signs, where $\operatorname{sgn}(a)=-1,0$, or 1 if $a<0,=0$, or $>0$.

$C$ and $S$ are normalized: $-1 \leqslant C(t) \leqslant 1$ and $-1 \leqslant S(t) \leqslant 1$ hold for all t. $C(t)=1$ or -1 if $x_{1}\left(t+\tau_{k}\right)=c x_{2}\left(t+\tau_{k}\right)$ with constant $c>0$ or $<0$ for all $k=1, \ldots, l$. Analogously, $S(t)=1$ or -1 if $x_{1}\left(t+\tau_{k}\right)$ and $x_{2}\left(t+\tau_{k}\right)$ have either the same sign for all $k=1, \ldots, l$ or different signs for all $k=1, \ldots, l . S(t)=0$ if at least one of the responses vanishes at time $t$ for all $k$ $=1, \ldots, l$.

\section{TRANSMISSION OF CT AVERAGED RESPONSES}

\section{A. Transmission of resets}

This section focuses on how a reset of oscillator 1 is transmitted to oscillator 2. For this, I choose the simplest case, where the two oscillators are 1:1 coupled, i.e., $n=m$ $=1$ in Eqs. (3) and (4). I assume that the coupling is strong enough compared to the noise amplitude $D$, so that without stimulation the two oscillators spontaneously synchronize in phase [Figs. 2(k)-2(m)]. The stimulus of oscillator 1 is modeled by $S\left(\psi_{1}\right)=I \cos \psi_{1}$. The stimulation intensity $I$ is large compared to both coupling strength $K$ and noise amplitude $D(K \ll I, D \ll I)$. Therefore oscillator 1 is quickly reset by the strong stimulus: $\phi_{1}$ is shifted close to $\phi_{1}^{\text {stat }} \approx 0.36$ [Fig. 2(a)]. This reset is reflected by an increase of the resetting index $\rho_{1}$ from Eq. (20) [Fig. 2(b)] and the entropy based index $\mu_{1}$ from Eq. (25) [Fig. 2(c)]. The CT averaged signal $\bar{x}_{1}$ from Eq. (1) vanishes during the prestimulus period because of the randomized stimulus administration [Fig. 2(e)]. The reset leads to an oscillatory $\mathrm{CT}$ averaged signal $\bar{x}_{1}$, which relaxes to zero due to noise.

The stimulus of oscillator 1 perturbs the strong synchronization between both oscillators. Because of the coupling between the two oscillators, the phase of oscillator 2 gets adapted to the phase of oscillator 1 within roughly a period. Before the reset of oscillator 2 reaches its maximum, the two oscillators undergo a transient epoch of desynchronization and resynchronization [Figs. 2(k)-2(m)]: As soon as both oscillators are fully resynchronized, the reset of oscillator 2 is maximal [Figs. 2(g) and 2(h)]. In this way the reset of oscillator 1 is transmitted onto oscillator 2 via the coupling. The reset of oscillator 2 corresponds to an increase of the resetting index $\rho_{2}$ [Fig. 2(g)] and the entropy based index $\mu_{2}$ 
(a)

(b)
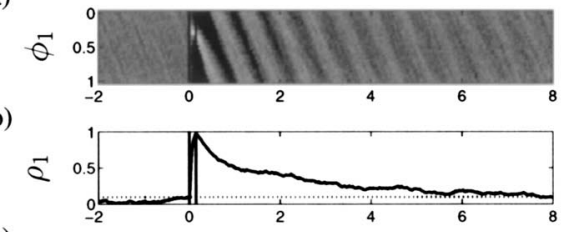

(c)

(d)

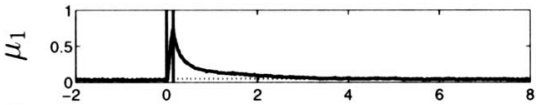

(e)

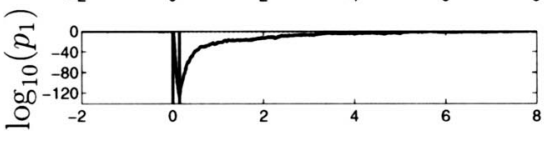

(f)
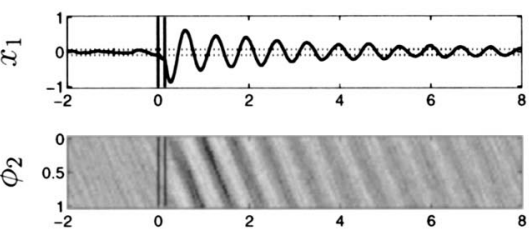

(g)

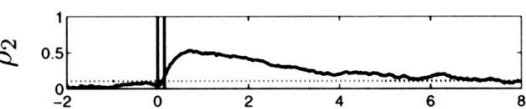

(h)

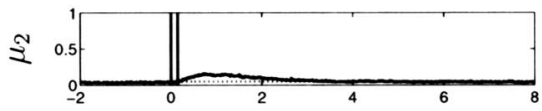

(i)

(j)
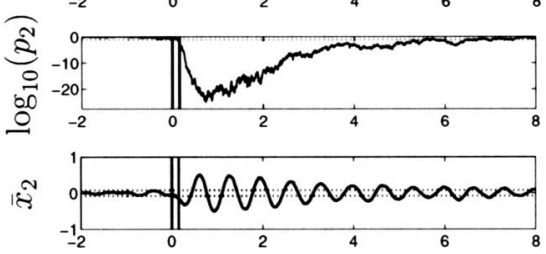

(k)

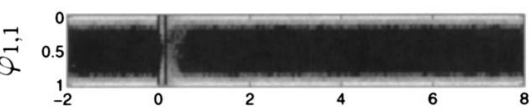

(l)

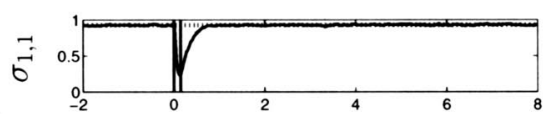

(m)

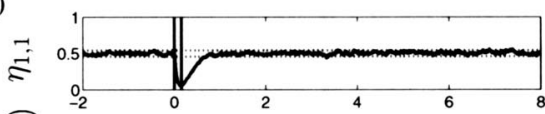

(n) $=$

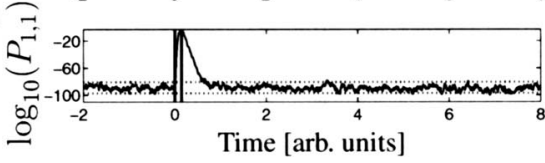

[Fig. 2(h)] and causes an oscillatory CT averaged signal $\bar{x}_{2}$, which vanishes in the course of the poststimulus period because of noise [Fig. 2(j)].

Let me compare the CT distribution of phase $\phi_{1}$ and the corresponding indices $\rho_{1}$ and $\mu_{1}$ [Figs. 2(a)-2(c)] with the CT distribution of phase $\phi_{2}$ and the corresponding indices $\rho_{2}$ and $\mu_{2}$ [Figs. 2(f)-2(h)]: The reset of oscillator 2 occurs by transmission via the coupling, and is delayed and less pronounced compared to the reset of oscillator 1. Furthermore, the reset of oscillator 2 appears after the stimulus-locked desynchronization [compare Figs. 2(g) and 2(1)].

\section{B. Cross-trial Kuiper test}

The reset of oscillator 1 shows up as an increase of the resetting index $\rho_{1}$ from Eq. (20) [Fig. 2(b)] and the entropy
FIG. 2. A strong stimulus $S\left(\psi_{1}\right)=I \cos \psi_{1}$ from Eq. (3) causes a reset of oscillator 1 which is transmitted to oscillator 2. CT distributions from Eq. (17) are shown as time-dependent histograms of $\phi_{j}$ and $\varphi_{1,1}$ calculated across trials for each time $t$ relative to stimulus onset within the time window $\left[t_{a}, t_{b}\right]:\left\{\phi_{1}\left(t+\tau_{k}\right)\right\}_{k=1, \ldots, l}$ in (a), $\left\{\phi_{2}\left(t+\tau_{k}\right)\right\}_{k=1, \ldots, l}$ in (f), and $\left\{\varphi_{1,1}\left(t+\tau_{k}\right)\right\}_{k=1, \ldots, l}$ in (k) (0 is black and maximal values are white). Indices from Eqs. (19)-(26) and CT averaged signals from Eq. (1): $\rho_{1}$ in (b), $\mu_{1}$ in (c), $\bar{x}_{1}$ in (e), $\rho_{2}$ in $(\mathrm{g}), \mu_{2}$ in (h), $\bar{x}_{2}$ in (j), $\sigma_{1,1}$ in (l), and $\eta_{1,1}$ in (m). Base 10 logarithm of the $P$ values of the CT Kuiper test for randomness: $\log _{10}\left(p_{j}\right)$ from Eq. (39) for the CT distribution of the phase of oscillator $j$ in (d) and (i); $\log _{10}\left(P_{n, m}\right)$ from Eq. (40) for the CT distribution of the $n: m$ phase difference in (n). Onset (at $t=0)$ and offset of the stimulus of oscillator 1 are indicated by solid vertical lines. Prestimulus interval $-t<0$, poststimulus interval $-t>0$. Significance levels: Dotted lines in (b), (c), (g), and (h) denote the 99th percentile of the corresponding prestimulus distributions. In (d), (e), (i), (j), (l), (m), and (n) upper and lower dotted lines indicate the 99th and the first percentile of the corresponding prestimulus distribution in the interval $[-8,0[$. Note, a noninteresting part of the prestimulus range of the time window $\left[t_{a}, t_{b}\right]=[-8,8]$ is not shown for the sake of clarity, but used for the calculation of the significance levels. Parameters of Eqs. (3) and (4): $K=3.5, n=m=1$, $\omega_{1} /(2 \pi)=1.5, \omega_{2} /(2 \pi)=1.494, D=1, I=40, \theta=0, t_{\text {win }}=16$ [see Eq. (16)], stimulus duration $=0.15$, and number of stimuli $l=200$. Results are stable with respect to variations of $l$ between 50 and 2000 and more. based index $\mu_{1}$ from Eq. (25) [Fig. 2(c)]. The CT Kuiper test of the CT distribution of $\phi_{1}$ [Fig. 2(d)] yields the $P$ value $p_{1}$ from Eq. (39). Because of the randomized stimulus administration the prestimulus CT distribution of $\phi_{1}$ is uniform. Therefore, $p_{1}$ is close to 1 , i.e., $\log _{10}\left(p_{1}\right)$ attains values between 0 and -1 . The strong reset corresponds to $\log _{10}\left(p_{1}\right)$ $=-128.3$ at stimulus offset. This means that with a probability of $10^{-128.3}$ the CT distribution of $\phi_{1}$ at stimulus offset is a uniform distribution. In other words, the null hypothesis (uniform distribution) can be rejected: At stimulus offset the CT distribution differs significantly from a uniform distribution.

By which of the three measures, $\rho_{1}, \mu_{1}$, and $p_{1}$, is the duration of the resetting epoch detected appropriately? The three quantities reveal practically the same time as onset of 
the reset. However, the end and, hence, the duration of the reset differs. $p_{1}$ exceeds its first prestimulus percentile for $t>5.63$. While $\mu_{1}$ attains values below its 99th prestimulus percentile already for $t>3.37, \rho_{1}$ falls below its 99 th percentile only for $t>7.7$. According to the impression one gets from a visual inspection of the CT distribution of $\phi_{1}$ [Fig. 2(a)], the time courses of both the resetting index $\rho_{1}$ and the $P$ value $p_{1}$ of the CT Kuiper test reflect the duration of the reset better compared to the entropy based index $\mu_{1}$.

The reset of oscillator 2 is weaker, but nevertheless reliably detected by means of the $P$ value $p_{2}$ from Eq. (39) of the CT Kuiper test [Fig. 2(i)]. More precisely, the CT Kuiper test detects the reset as an epoch with a significantly nonuniform CT distribution of $\phi_{2}$. Again, the duration of the reset is better detected with the CT Kuiper test and with the resetting index $\rho_{2}$ [Fig. 2(g)] as opposed to the index $\mu_{2}$ [Fig. 2(h)].

The transient desynchronization is related to a decrease of the $1: 1$ synchronization index $\sigma_{1,1}$ from Eq. (19) and a decrease of the entropy based $1: 1$ stimulus-locking index $\eta_{1,1}$ from Eq. (26) below their first prestimulus percentile [Fig. 2(1)], respectively. Likewise, the CT Kuiper test detects the transient desynchronization. The strong synchronization during the prestimulus epoch is connected with a small $P$ value $P_{1,1}$ from Eq. (40) [Fig. 2(n)]: The null hypothesis (uniform distribution) is clearly rejected. In contrast, during the transient epoch of desynchronization the CT distribution of $\varphi_{1,1}$ gets more random, so that $\log _{10}\left(P_{1,1}\right)$ exceeds its 99th prestimulus percentile. As a consequence of the resynchronization, $\log _{10}\left(P_{1,1}\right)$ enters its prestimulus range again.

\section{Transmission time}

Let me consider how the transmission time of the reset, i.e., the time elapsing between the maximal reset of oscillator 1 and the maximal reset of oscillator 2 depends on different model parameters. I compare it with the transmission time of the CT average, i.e., the time between the maximum of the CT averaged signal of oscillator 1 and the maximum of the CT averaged signal of oscillator 2. I normalize both quantities by division by the mean period of the oscillators, $T$ $=4 \pi /\left(\omega_{1}+\omega_{2}\right)$, and obtain the normalized transmission time of the reset

$$
\Delta t_{\mathrm{re}}^{*}=\frac{t_{\mathrm{re}}^{(2)}-t_{\mathrm{re}}^{(1)}}{T}
$$

where $t_{\mathrm{re}}^{(j)}$ is the time at which the resetting index $\rho_{j}$ from Eq. (20) is maximal. The maximal value of the resetting index of oscillator $j$ is denoted by

$$
\hat{\rho}_{j}=\rho_{j}\left(t_{\mathrm{re}}^{(j)}\right) .
$$

Analogously, one obtains the normalized transmission time of the CT average

$$
\Delta t_{\mathrm{av}}^{*}=\frac{t_{\mathrm{av}}^{(2)}-t_{\mathrm{av}}^{(1)}}{T}
$$

with $t_{\mathrm{av}}^{(j)}$ being the time at which the CT averaged signal $\bar{x}_{j}$ from Eq. (1) is maximal. $\Delta t_{\mathrm{av}}^{*}$ corresponds to the standard method for the detection of transmission times, as used in medicine and biology $[6,7,10,11]$. The maximal value of the $\mathrm{CT}$ averaged signal of oscillator $j$ reads

$$
\hat{x}_{j}=\bar{x}_{j}\left(t_{\mathrm{av}}^{(j)}\right) .
$$

In this study I consider a stimulation term of lowest order, i.e., a stimulation mechanism modeled by trigonometric terms of first order:

$$
S\left(\psi_{1}\right)=I \cos \left(\psi_{1}+\chi\right),
$$

where $\chi$ is a constant.

First, I assume that $\chi$ from Eq. (48) as well as the shift term $\theta$ in the couplings in Eqs. (3) and (4) vanish: $\chi=\theta=0$. I study the impact of the coupling strength on the normalized transmission times, $\Delta t_{\mathrm{re}}^{*}$ from Eq. (44) and $\Delta t_{\mathrm{av}}^{*}$ from Eq. (46). With increasing coupling strength $K$ the normalized transmission time of the reset, $\Delta t_{\mathrm{re}}^{*}$, decreases gradually [Fig. 3(a)]. For values of $K$ greater than the intensity parameter $I$ from Eq. (48) ( $I=40$ in Fig. 3), $\Delta t_{\text {re }}^{*}$ finally converges to zero: The two oscillators then behave like one composite oscillator. This convergence is not shown in Fig. 3; rather I focus on phenomena occurring for weaker coupling, since the latter is more realistic for applications to biology and medicine.

Unlike $\Delta t_{\mathrm{re}}^{*}$, the normalized transmission time of the CT average $\Delta t_{\mathrm{av}}^{*}$ from Eq. (46) displays a discrete dependence on $K$ : Over the whole range of the coupling constant, i.e., for $0 \leqslant K \leqslant 15, \Delta t_{\mathrm{av}}^{*}$ takes only discrete values (except for minor fluctuations) 0 , 1 , and 2. With an increase of $K, \Delta t_{\mathrm{av}}^{*}$ jumps from 2 to 1 and finally to 0 , with an overlap of the different levels of $\Delta t_{\mathrm{av}}^{*}$.

To understand the discrete dependence of $\Delta t_{\mathrm{av}}^{*}$ on $K$, let me consider the time course of $\bar{x}_{j}$ for intermediate coupling $(K=2.02)$ and strong coupling $(K=11.3)$ [Figs. 4(a) and 4(b)]. According to their coupling mechanism, defined by Eqs. (3) and (4) with $\theta=0$, both oscillators spontaneously tend to synchronize in phase. A maximum of the CT averaged response $\bar{x}_{2}$ requires a maximum of the reset of oscillator 2, which, in turn, requires a complete in-phase resynchronization of the two oscillators [see discussion in the former section and Figs. 2(f) and 2(j)]. As a consequence of the particular form of the coupling, which induces an inphase synchronization, the maxima of $\bar{x}_{1}$ and $\bar{x}_{2}$ are separated by multiples of their period $T=4 \pi /\left(\omega_{1}+\omega_{2}\right)$. The quicker the resynchronization takes place, the stronger the coupling is. Accordingly, for strong coupling the maxima of $\bar{x}_{1}$ and $\bar{x}_{2}$ coincide, and $\Delta t_{\mathrm{av}}^{*}=0$. In contrast, for intermediate coupling it takes $\bar{x}_{2}$ one more period to attain its maximum: $\Delta t_{\mathrm{av}}^{*}=1$.

The fact that $\Delta t_{\mathrm{re}}^{*}$ depends on $K$ gradually, whereas $\Delta t_{\mathrm{av}}^{*}$ depends on $K$ in a discrete way, is stable with respect to variations of the number of trials $l$. While $l$ equals 800 in Fig. 3(a), $l$ equals 200 in Fig. 3(c). With a smaller number of trials $l$ the amplitude of the variations of $\Delta t_{\mathrm{re}}^{*}$ around the curve obtained for $l=800$ increases, especially for small coupling strength. In contrast, for smaller $l$ the range of $K$ with overlapping levels of $\Delta t_{\mathrm{av}}^{*}$ increases, the levels itself get more noisy, and even higher levels of $\Delta t_{\mathrm{av}}^{*}$ are added: For one value of $K$ one gets $\Delta t_{\mathrm{av}}^{*}=3$. 
(a)

(c)
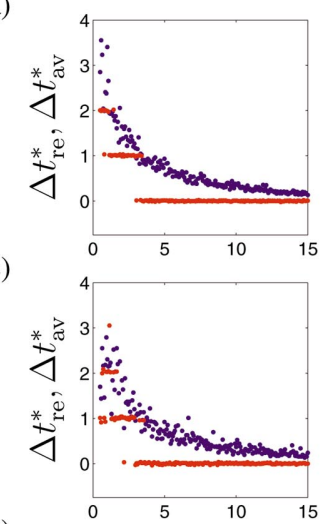

(e)

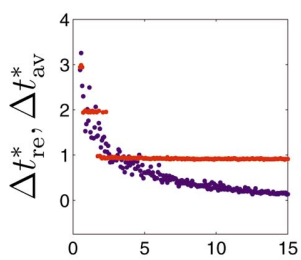

$(\mathrm{g})$

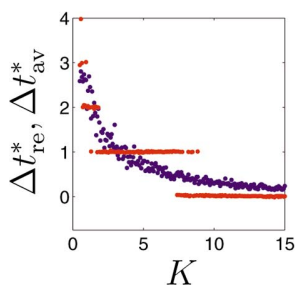

(b)

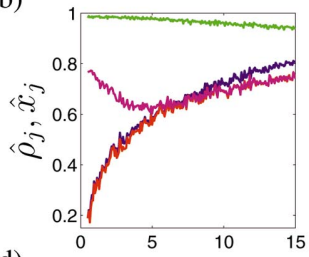

(d)

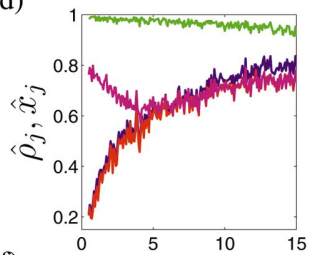

(f)

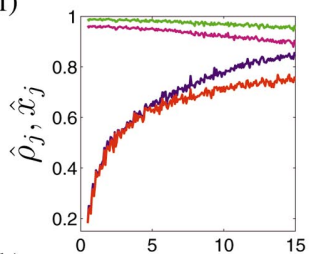

(h)

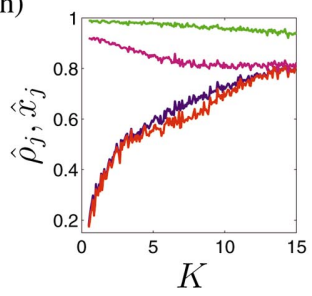

(i)

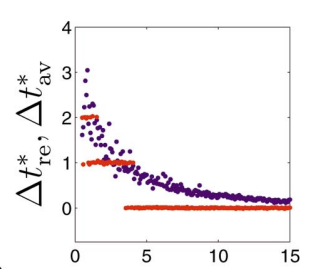

(k)

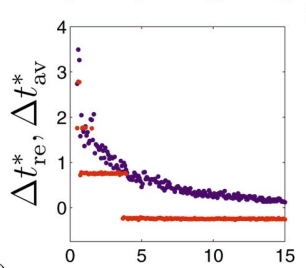

(m)

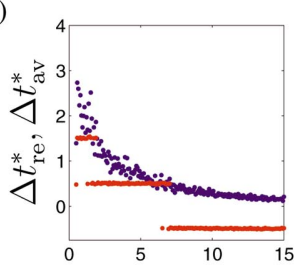

(o)

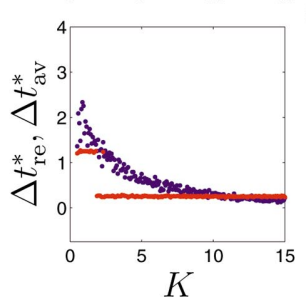

(j)
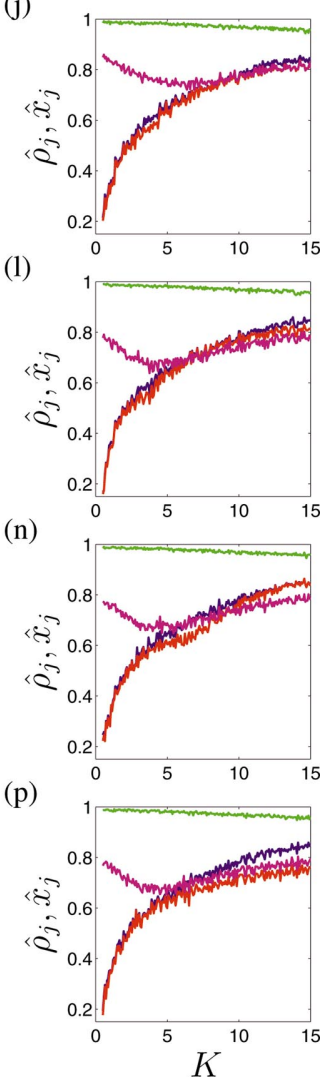

FIG. 3. (Color) In series of simulations the coupling strength $K$ of Eqs. (3) and (4) is varied between 0.5 and 15 . The stimulation parameter $\chi$ of $S\left(\psi_{1}\right)=I \cos \left(\psi_{1}+\chi\right)$ from Eq. (48) and the phase shift $\theta$ of the coupling terms of Eqs. (3) and (4) are varied between different series. Normalized transmission time of the reset $\Delta t_{\mathrm{re}}^{*}$ from Eq. (44) (blue dots) and normalized transmission time of the CT average $\Delta t_{\mathrm{av}}^{*}$ from Eq. (46) (red dots) are displayed in (a), (c), (e), (g), (i), (k), (m), and (o). The maximal values of the resetting index of both oscillators, $\hat{\rho}_{1}$ (green) and $\hat{\rho}_{2}$ (blue) from Eq. (45), and the maximal value of the CT averaged signal of both oscillators, $\hat{x}_{1}$ (magenta) and $\hat{x}_{2}$ (red) from Eq. (47), are plotted in (b), (d), (f), (h), (j), (l), (n), and (p). (a)-(j) Variation of the stimulation parameter $\chi$, while coupling parameter $\theta$ is constantly equal to 0: In (b) number of trials $l$ equals 200, whereas in all other plots $l=800$. Stimulation parameter $\chi=0$ in (a)-(d), $\chi$ $=\pi / 2$ in (e),(f), $\chi=\pi$ in (g),(h), and $\chi=3 \pi / 2$ in (i),(j). (k)-(p) Variation of the coupling parameter $\theta$, with constant stimulation parameter $\chi=0: \theta=\pi / 2$ in (k),(l), $\theta=\pi$, in (m),(n), and $\theta=3 \pi / 2$ in (o),(p).

\section{Impact of the stimulation parameter $\chi$}

Does the phase to which oscillator 1 is reset by the stimulus influence the normalized transmission times $\Delta t_{\mathrm{re}}^{*}$ and $\Delta t_{\mathrm{av}}^{*}$ ? Put otherwise, how does the stimulation parameter $\chi$ from Eq. (48) modify the normalized transmission times? To study the impact of the stimulation mechanism, the coupling is kept constant, i.e., $\theta=0$ in Eqs. (3) and (4). The parameter $\chi$ in the stimulation mechanism $S\left(\psi_{1}\right)=I \cos \left(\psi_{1}+\chi\right)$ from Eq. (48) determines the phase to which oscillator 1 is reset at the end of the stimulation. A stimulus modelled by $S\left(\psi_{1}\right)$ $=I \cos \left(\psi_{1}+k \pi / 2\right)$ resets the phase of oscillator 1 to $\left[\phi_{1}^{\text {stat }}\right.$ $-k \pi / 2] \bmod 1$, where $\phi_{1}^{\text {stat }} \approx 0.36$, and $k=0,1,2,3$ (see Fig. 2(a)) and Ref. [5]). Note that $\phi_{j}(t)=\psi_{j}(t) /(2 \pi) \bmod 1$ according to Eq. (14). I determine how the normalized transmission times depend on $K$ for different values of $\chi: \chi=0$ in Fig. 3(a), $\chi=\pi / 2$ in Fig. 3(e), $\chi=\pi$ in Fig. $3(\mathrm{~g})$, and $\chi=3 \pi / 2$ in Fig. 3(i). All other parameters in Figs. 3(e), 3(g), and 3(i) are identical to those in Fig. 3(a).

Except for minor fluctuations the dependence of $\Delta t_{\text {re }}^{*}$ on $K$ is not modified by the different values of $\chi$. In contrast, the differences between the $\Delta t_{\mathrm{av}}^{*}$ vs $K$ curve for different values of $\chi$ are more pronounced. $\Delta t_{\mathrm{av}}^{*}$ may attain integer values between 0 and 4 . However, the coupling range, where the different levels of $\Delta t_{\mathrm{av}}^{*}$ occur, as well as the range of the discrete levels of $\Delta t_{\mathrm{av}}^{*}$ strongly depend on the parameter $\chi$. Only for $\chi=\pi$ the normalized transmission time $\Delta t_{\mathrm{av}}^{*}$ attains values up to 4 , whereas for $\chi=\pi$ no zero values of $\Delta t_{\mathrm{av}}^{*}$ occur. The latter is due to $\bar{x}_{1}$ having its maximum directly at the end of the stimulation for $\chi=\pi$. For the range of $K$ considered here, the resynchronization of the two oscillators takes longer than the short time distance between the end of the stimulation and the occurrence of $\bar{x}_{1}$ 's maximum.

In summary, the dependence of $\Delta t_{\text {re }}^{*}$ on $K$ is basically invariant with respect to variations of $\chi$. The dependence of $\Delta t_{\mathrm{av}}^{*}$ on $K$ remains discrete, where the concrete shape of this curve undergoes changes depending on the value of $\chi$. However, one important feature of the $\Delta t_{\mathrm{av}}^{*}$ vs $K$ curve remains invariant with respect to variations of $\chi: \Delta t_{\mathrm{av}}^{*}$ corresponds to the phase difference $\varphi_{1,1}^{\mathrm{s}}$ between the two oscillators in the stable synchronized state plus an integer $k$, where $k$ represents the number of periods of length $T$, which are left over after dividing $k T$ by $T$ in Eq. (46). Accordingly, for in-phase 
(a)

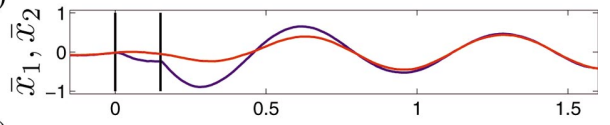

(b)

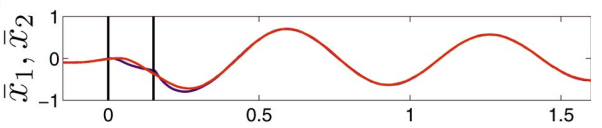

(c)

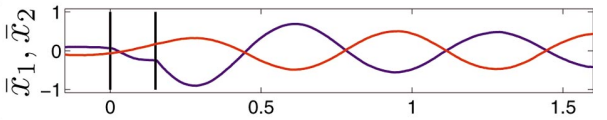

(d)

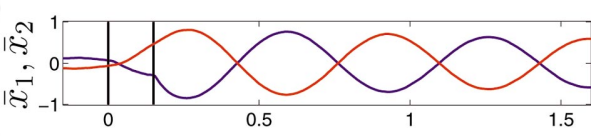

(e)

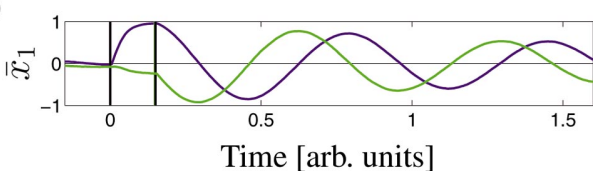

FIG. 4. (Color) (a)-(d) Time course of the CT averaged signals $\bar{x}_{1}$ (blue line) and $\bar{x}_{2}$ (red line) from Eq. (1) belonging to selected simulations of Fig. 3. (a) and (b) belong to Fig. 3(a), whereas (c) and (d) belong to Fig. 3(m). Coupling strengths: $K=2.02$ (a), $K$ $=11.3$ (b), $K=2.6$ (c), and $K=11.3$ (d). (e) $\bar{x}_{1}$ belonging to the simulations of Fig. 3(b) (green line) and of Fig. 3(f) (blue line) with $K=0.5$ each.

coupling with vanishing detuning $\Delta t_{\mathrm{av}}^{*}$ may attain the values $0,1,2$, etc., depending on the coupling strength $K$.

\section{Impact of the coupling parameter $\theta$}

In this Section I focus on how $\Delta t_{\mathrm{re}}^{*}$ and $\Delta t_{\mathrm{av}}^{*}$ are influenced by the coupling parameter $\theta$. For this, the stimulation parameter $\chi$ in the stimulation mechanism $S\left(\psi_{1}\right)=I \cos \left(\psi_{1}+\chi\right)$ from Eq. (48) is constantly set to zero. The dependence of the normalized transmission times on $K$ is plotted for different values of $\theta: \theta=0$ in Fig. 3(a), $\theta=\pi / 2$ in Fig. 3(k), $\theta=\pi$ in Fig. 3(m), and $\theta=3 \pi / 2$ in Fig. 3(o). All other parameters in Figs. 3(k), 3(m), and 3(o) are identical to those in Fig. 3(a).

Neglecting minor fluctuations, the dependence of $\Delta t_{\mathrm{re}}^{*}$ on $K$ is invariant with respect to variations of $\theta$. In contrast, $\Delta t_{\mathrm{av}}^{*}$ crucially depends on $\theta$. From the coupling mechanism it follows that the phase difference in the synchronized state reads $\varphi_{1,1}=0$ in Fig. 3(a), $\varphi_{1,1}=0.75$ in Fig. 3(k), $\varphi_{1,1}=0.5$ in Fig. $3(\mathrm{~m})$, and $\varphi_{1,1}=0.25$ in Fig. 3(o). Correspondingly, for $\theta=0$ we get $\Delta t_{\mathrm{av}}^{*}=0,1$, or 2 [Fig. 3(a)]. For $\theta=\pi / 2, \Delta t_{\mathrm{av}}^{*}$ attains the values $k+0.75$ with $k=-1,0,1,2$ [Fig. 3(k)]. For $\theta=\pi$, $\Delta t_{\mathrm{av}}^{*}$ takes the values $k+0.5$ with $k=-1,0,1$ [Fig. 3(m)]. For $\theta=3 \pi / 2$ we get $\Delta t_{\mathrm{av}}^{*}=k+0.25$ with $k=0,1$ [Fig. 3(o)]. In summary,

$$
\Delta t_{\mathrm{av}}^{*}=k+\frac{\theta}{2 \pi}
$$

with integer $k$, and, hence, $\Delta t_{\mathrm{av}}^{*}$ directly corresponds to the phase difference between the two oscillators spontaneously emerging during the poststimulus process of resynchronization according to the coupling mechanism plus an integer $k$.
The integer $k$ represents a number of periods of length $k T$ which remains after the normalization, i.e., after division by the period $T$ in Eq. (46).

The occurrence of negative values of $\Delta t_{\mathrm{re}}^{*}$ is illustrated in Figs. 4(c) and 4(d), which show the time course of $\bar{x}_{j}$ for intermediate coupling $(K=2.6)$ and strong coupling $(K$ $=11.3$ ), respectively. The simulations shown in Figs. 4(c) and 4(d) belong to the series of simulations displayed in Fig. $3(\mathrm{~m})$. As $\theta=\pi$, both oscillators spontaneously tend to synchronize in antiphase. Therefore the maxima of $\bar{x}_{1}$ and $\bar{x}_{2}$ are shifted in time by $T / 2+k T$, where $T=4 \pi /\left(\omega_{1}+\omega_{2}\right)$ is the period of the oscillations and $k$ is an integer. For smaller or larger coupling strength $K$, the process of the poststimulus resynchronization takes a longer or shorter time, and, hence, the maximal reset of oscillator 2, combined with a maximum of $\bar{x}_{2}$, appears at a later or earlier time. $\bar{x}_{1}$ has its minimum directly after the stimulation. Thus, for sufficiently large $K$ the maximum of $\bar{x}_{2}$ occurs before the maximum of $\bar{x}_{1}$ [Fig. 4(d)].

The variation of the coupling parameter $\theta$ illustrates that $\Delta t_{\mathrm{av}}^{*}$ corresponds to the phase difference $\varphi_{1,1}^{\mathrm{s}}$ between the two oscillators in the stable synchronized state. To understand the dependence of $\varphi_{1,1}^{\mathrm{s}}$ on model parameters, let me consider Eq. (13), which shows how the coupling mechanism, i.e., the values of $n$ and $m$, the coupling strength $K$, the detuning $\gamma=n \omega_{1}-m \omega_{2}$, and the coupling parameter $\theta$ determine the value of the stable fixed point $\Phi_{n, m}^{\mathrm{s}}$. I recall that $\varphi_{1,1}$ and $\Phi_{1,1}$ correspond to each other according to Eqs. (8) and (15). From Eq. (13) it follows that by increasing $\theta$ from 0 to $2 \pi, \varphi_{1,1}^{s}$ is shifted once through a cycle.

\section{Strength of reset vs amplitude of CT averaged signal}

The maximal value of the resetting index of the $j$ th oscillator is denoted by $\hat{\rho}_{j}=\rho_{j}\left(t_{\mathrm{re}}^{(j)}\right)$, whereas the maximum of the CT averaged signal of the $j$ th oscillator reads $\hat{x}_{j}=\bar{x}_{j}\left(t_{\mathrm{av}}^{(j)}\right)$ [see Eqs. (45) and (47)]. According to Eq. (48) the stimulation mechanism is given by $S\left(\psi_{1}\right)=I \cos \left(\psi_{1}+\chi\right)$. Figure 3 shows the impact of the stimulation parameter $\chi$ and the coupling parameter $\theta$ on $\hat{\rho}_{j}$ and $\hat{x}_{j}$.

Except for minor variations the dependence of $\hat{\rho}_{1}$ and $\hat{\rho}_{2}$ on the coupling strength $K$ hardly changes with respect to variations of $\chi$ and $\theta$. This also holds for a smaller number of trials $[l=200$ in Fig. 3(d), compared to $l=800$ in all other plots of Fig. 3]. With an increase of the coupling strength $K$ the strength of the transmission and, thus, the strength of the transmitted reset of oscillator 2 increases: $\hat{\rho}_{2}$ strongly increases with an increase of $K$. In contrast, with an increase of the coupling strength $K$, the reset of oscillator 1 , as detected by $\hat{\rho}_{1}$, moderately decreases. For sufficiently large coupling strength $K$, i.e., with $K$ greater than the intensity parameter $I$ ( $I=40$ in Fig. 3 ), $\hat{\rho}_{1}$ and $\hat{\rho}_{2}$ finally converge: The two oscillators are then tightly coupled and behave like one giant oscillator. The reset of this composite oscillator is less pronounced compared to the reset of a single oscillator (see $\hat{\rho}_{1}$ ). The range of the coupling strength in Fig. 3 is restricted to values up to 15 , in order to stick to values that are realistic in the context of models in biology and medicine. 
Since a reset leads to a nonvanishing CT averaged signal $\bar{x}_{j}$ from Eq. (1) [see Figs. 2(a)-2(h)], with an increase of $K$ the CT averaged response and, thus, $\hat{x}_{2}$ increases (Fig. 3). The shape of the $\hat{x}_{2}$ vs $K$ curve depends on the stimulation parameter $\chi$ and the coupling parameter $\theta$. These variations are moderate compared to how strongly the shape of the $\hat{x}_{1}$ vs $K$ curve depends, in particular, on the stimulation parameter $\chi$ [see, e.g., Figs. 3(b) and 3(f)]. This is due to the parameter $\chi$ determining to which phase oscillator 1 is reset at the end of the stimulation. For suitable $\chi$, a maximum of $\hat{x}_{1}$ occurs directly at the end of the stimulation [Fig. 4(e), blue line]. In contrast, the later $\hat{x}_{1}$ 's maximum occurs after the stimulation, the smaller is its amplitude [Fig. 4(e), green line]. For sufficiently large coupling strength $K$ the two oscillators act as one joint oscillator, so that finally $\hat{x}_{1}$ and $\hat{x}_{2}$ converge (Fig. 3).

\section{VI. $n: m$ COUPLING}

Let me now turn to $n: m$ coupling, where $n \neq 1$ and/or $m$ $\neq 1$ (and $n \neq m$ ) in Eqs. (3) and (4). Again, only oscillator 1 is stimulated, and the perturbation is transmitted to oscillator 2. However, depending on the interplay of stimulation mechanism, modeled by $S\left(\psi_{1}\right)$ [e.g., Eq. (48)], and $n: m$ coupling, oscillator 1 and/or oscillator 2 do not exhibit a CT averaged response. In other words, the transmission of the stimulus' action escapes detection when cross-trial averaging is used. In contrast, the cross-trial stochastic phase resetting analysis from Sec. III reliably detects the transient processes under consideration.

All of the dynamical phenomena presented in Secs. VII-IX are due to a major difference between 1:1 coupling and $n: m$ coupling: As explained in Sec. II B, in case of the $1: 1$ coupling Eq. (9), the evolution equation of the $1: 1$ phase difference $\Phi_{1,1}$, has only one stable fixed point $\Phi_{1,1}^{\mathrm{s}}$ modulo $2 \pi$ (for vanishing noise and $K>\gamma$ ). This stable fixed point, in turn, is related to only one stable solution of the phases $\psi_{1}$ and $\psi_{2}$ (modulo $2 \pi$ ). In contrast, with $n: m$ coupling a bistability and multistability comes into play: Also for $n: m$ coupling there is only one stable fixed point $\Phi_{n, m}^{\mathrm{s}}$ modulo $2 \pi$ (for vanishing noise and $K>\gamma$ ), which is due to the simple in-phase coupling of lowest order, $\sin \left(n \psi_{1}-m \psi_{2}+\theta\right)$ and $\sin \left(m \psi_{2}-n \psi_{1}-\theta\right)$ in Eqs. (3) and (4) (see Sec. II B). However, the stable fixed point $\Phi_{n, m}^{\mathrm{s}}$ now corresponds to more than only one stable solution of the phases $\psi_{1}$ and $\psi_{2}$ (modulo $2 \pi$ ). The number of different stable synchronized states depends on the values of $n$ and $m$ (see Sec. II B). Consequently, after stimulation the two $n: m$ coupled oscillators may relax to different synchronized states.

\section{NO TRANSMISSION OF THE CT AVERAGED RESPONSE}

\section{A. 1:2 coupling}

In this section I demonstrate how by transmission a reset of oscillator 1 causes an antiphase CT response clustering of oscillator 2. To this end, I consider 1:2 coupled oscillators with eigenfrequencies $\omega_{1} \approx 2 \omega_{2}$ (Fig. 5). A strong stimulus $S\left(\psi_{1}\right)=I \cos \psi_{1}$ resets oscillator 1 [Fig. 5(a)]. This is re- flected by the time course of the resetting index $\rho_{1}$ from Eq. (20) [Fig. 5(b)] and the entropy based index $\mu_{1}$ from Eq. (25) [Fig. 5(e)]. Additionally, the reset is illustrated by means of a phase resetting curve $[3,5]$ showing $\phi_{1}^{\mathrm{E}}$ plotted over $\phi_{1}^{\mathrm{B}}$ for all $l$ trials, where $\phi_{1}^{\mathrm{B}}$ and $\phi_{1}^{\mathrm{E}}$ denote the phase of oscillator 1 at the beginning and at the end of the stimulation, respectively [Fig. 6(a)]. Nearly irrespective of the initial phase $\phi_{1}^{\mathrm{B}}$, oscillator 1 is always reset close to 0.3 . Such a horizontal phase resetting curve is a signature of a strong reset $[3,5]$.

In contrast, the phase of oscillator 2 is not stereotypically reset to one phase value. Rather, oscillator 2 adapts its phase to the phase of oscillator 1. By doing this, as described in Sec. VI, oscillator 2 may tend to one of two different, antiphase stable states. This is illustrated by means of a phase resetting curve with $\phi_{2}^{\mathrm{C}}$ being plotted over $\phi_{2}^{\mathrm{B}}$. $\phi_{2}^{\mathrm{C}}$ is the phase of oscillator 2 at the time when the antiphase CT response clustering is maximal, i.e., when the antiphase CT response clustering index $\alpha_{2}$ from Eq. (21) is maximal [at time $t_{\mathrm{cl}}^{(2)}=0.56$, Fig. $\left.5(\mathrm{~m})\right] . \phi_{2}^{\mathrm{B}}$ is the phase of oscillator 2 at the beginning of the stimulation. For $0.3<\phi_{2}^{\mathrm{B}}<0.78, \phi_{2}^{\mathrm{C}}$ predominantly lies around 0.95 (modulo 1 ), whereas $\phi_{2}^{\mathrm{C}}$ attains values around 0.45 else. Put otherwise, depending on the initial conditions oscillator 2 switches between two different antiphase responses across trials. The antiphase CT response clustering is detected with the antiphase CT response clustering index $\alpha_{2}$ [Fig. 5(m)] and, to a certain extent, also with the entropy based index $\mu_{2}$ [Fig. 5(n)], whereas it escapes detection with the resetting index $\rho_{2}$ [Fig. $5(\mathrm{k})]$.

Before stimulation both oscillators are synchronized [Fig. $5(\mathrm{~h})$ ]. The reset of oscillator 1 perturbs the synchronization, so that a transient stimulus-locked desynchronization occurs: Both the 1:2 synchronization index $\sigma_{1,2}$ from Eq. (19) and the related entropy based index $\eta_{1,2}$ from Eq. (26) fall below the prestimulus baseline, i.e., below the first percentile of the corresponding CT distribution [Figs. 5(i) and 5(q)]. As soon as oscillator 2 has adapted its phase to the phase of oscillator 1 , the antiphase CT response clustering is maximal [Fig. $5(\mathrm{~m})]$, and the stimulus-locked synchronization has reached its prestimulus level [Figs. 5(i) and 5(q)].

Let us study the transmission time elapsing between the maximal reset of oscillator 1 and the maximal CT response clustering of oscillator 2 . Normalizing this quantity by division by the mean period of the oscillators, $T=4 \pi /\left(\omega_{1}+\omega_{2}\right)$, we obtain the normalized transmission time between the reset of oscillator 1 and the CT response clustering of oscillator 2,

$$
\Delta t_{\mathrm{cl}-\mathrm{re}}^{*}=\frac{t_{\mathrm{cl}}^{(2)}-t_{\mathrm{re}}^{(1)}}{T}
$$

where $t_{\mathrm{re}}^{(1)}$ is the time at which the resetting index $\rho_{1}$ from Eq. (20) is maximal, whereas $t_{\mathrm{cl}}^{(2)}$ is the time at which the antiphase CT response clustering index $\alpha_{2}$ from Eq. (21) is maximal.

To focus on the impact of the coupling on the transmission of the stimulus' effect, I determine how $\Delta t_{\text {cl-re }}^{*}$ changes with an increase of the coupling strength $K$, while all other model parameters are kept constant. With an increase of the 
(a)

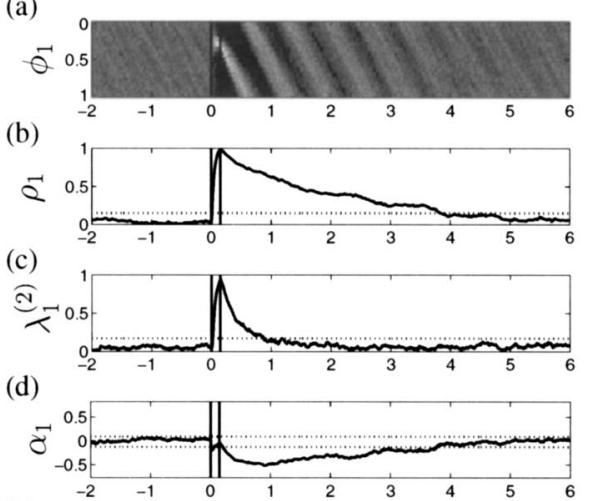

(e)
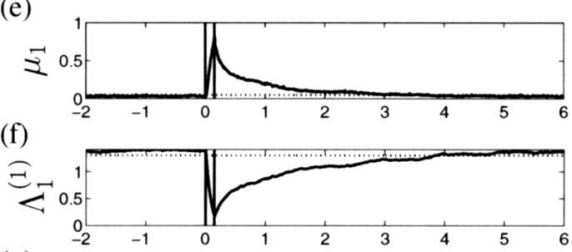

(g)

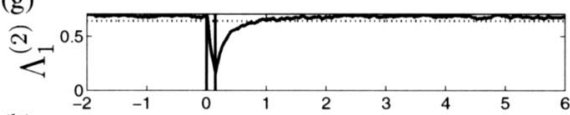

(h)

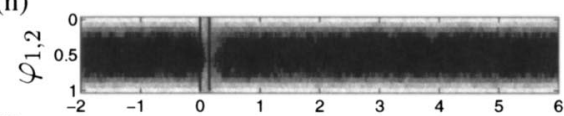

(i)

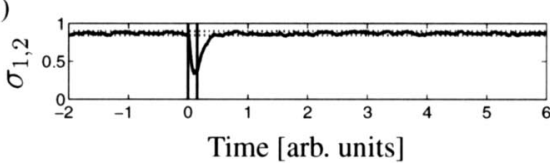

(j)

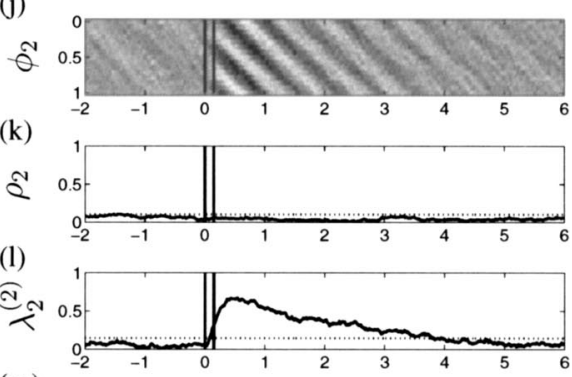

(m)

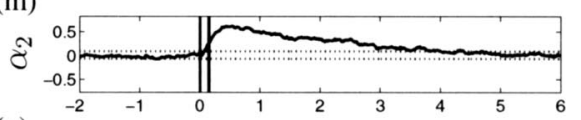

(n)
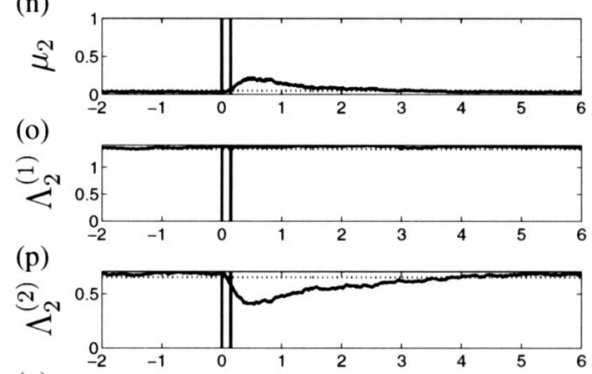

(q)

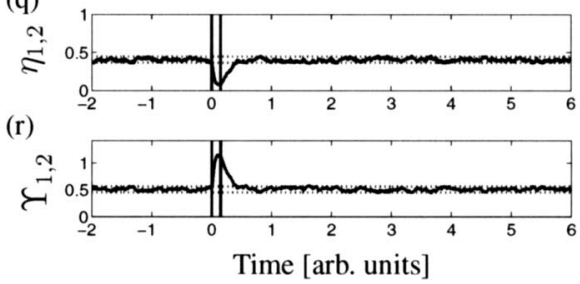

FIG. 5. A strong stimulus $S\left(\psi_{1}\right)=I \cos \psi_{1}$ from Eq. (3) causes a reset of oscillator 1 which, being transmitted, shows up as antiphase CT response clustering of oscillator 2. CT distributions from Eq. (17): $\left\{\phi_{1}\left(t+\tau_{k}\right)\right\}_{k=1, \ldots, l}$ in (a), $\left\{\varphi_{1,2}\left(t+\tau_{k}\right)\right\}_{k=1, \ldots, l}$ in (h), and $\left\{\phi_{2}(t\right.$ $\left.\left.+\tau_{k}\right)\right\}_{k=1, \ldots, l}$ in (j) (0 is black and maximal values are white). Quantities from Eqs. (18)-(26): $\rho_{1}$ in (b), $\lambda_{1}^{(2)}$ in (c), $\alpha_{1}$ in (d), $\mu_{1}$ in (e), $\Lambda_{1}^{(1)}$ in (f), $\Lambda_{1}^{(2)}$ in (g), $\sigma_{1,2}$ in (i), $\rho_{2}$ in (k), $\lambda_{2}^{(2)}$ in (1), $\alpha_{2}$ in (m), $\mu_{2}$ in (n), $\Lambda_{2}^{(1)}$ in (o), $\Lambda_{2}^{(2)}$ in (p), $\eta_{1,2}$ in (q), and $Y_{1,2}$ in (r). Same format as in Fig. 2. Significance levels: Dotted lines in (b), (c), (e), (k), (l), and (n) denote the 99th percentile of the corresponding prestimulus distributions. Dotted lines in (f), (g), (o), and (p) denote the first percentile of the corresponding prestimulus distributions. In (d), (i) and (m), (q), and (r) upper and lower dotted lines indicate the 99th and the first percentile of the corresponding prestimulus distribution in the interval $\left[-8,0\left[\right.\right.$. Prestimulus time window used for calculating the significance levels: $\left[t_{a}, 0[=[-8,0[\right.$. Parameters of Eqs. (3) and (4): $K=3.5, n=1$, $m=2, \omega_{1} /(2 \pi)=1.5, \omega_{2} /(2 \pi)=0.747, D=1, I=40, \theta=0 t_{\text {win }}=16$ [see Eq. (16)], stimulus duration $=0.15$, and number of stimuli $l=200$. Results are stable with respect to variations of $l$ between 50 and 2000 and more.

coupling strength $K, \Delta t_{\text {cl-re }}^{*}$ decreases gradually [Fig. 6(c), compare Fig. 3]. A quantity comparable to $\Delta t_{\mathrm{av}}^{*}$ from Eq. (46) cannot be used here, since the CT averaged response of oscillator 2 vanishes due to the antiphase CT response clustering [Fig. 7(c)].

To assess how the extent of the reset and of the CT response clustering vary with variations of the coupling strength, I introduce the maximal value of $\alpha_{2}$, the antiphase CT clustering index of oscillator 2 from Eq. (21), by

$$
\hat{\alpha}_{2}=\alpha_{2}\left(t_{\mathrm{cl}}^{(2)}\right) \text {. }
$$

Additionally I use $\hat{\rho}_{1}$, the maximal value of the resetting index of oscillator 1 from Eq. (45). With an increase of $K$ the strength of the reset of oscillator 1 slightly decreases, whereas the strength of the antiphase CT response clustering strongly increases [Fig. 6(d)].

\section{B. Mean angular deviation}

Based on techniques from circular statistics [33], in Sec. III the CT mean angular deviation $\Lambda_{j}^{(1)}(t)$ for a unimodal CT distribution $\left\{\phi_{j}\left(t+\tau_{k}\right)\right\}_{k=1, \ldots, l}$ at time $t$ and the CT mean angular deviation $\Lambda_{j}^{(2)}(t)$ for an antiphase bimodal CT distribution $\left\{\phi_{j}\left(t+\tau_{k}\right)\right\}_{k=1, \ldots, l}$ at time $t$ have been introduced as additional measures for the stimulus-locking indices $\lambda_{j}^{(1)}(t)$ and $\lambda_{j}^{(2)}(t)$ [Eqs. (18), (20), and (23)]. A strong reset corresponds to large values of the resetting index $\rho_{j}(t)=\lambda_{j}^{(1)}(t)$ and small values of the CT mean angular deviation $\Lambda_{j}^{(1)}(t)$. Absence of a reset is related to small $\rho_{j}(t)$ and large $\Lambda_{j}^{(1)}(t)$. This relationship between $\rho_{j}$ and $\Lambda_{j}^{(1)}$ is reflected by the time courses in Figs. 5(b) and 5(f) as well as Figs. 5(k) and 5(o).

According to Eqs. (19) and (24), the CT mean angular deviation $\Upsilon_{n, m}(t)$ for a unimodal CT distribution $\left\{\varphi_{n, m}(t\right.$ $\left.\left.+\tau_{k}\right)\right\}_{k=1, \ldots, l}$ at time $t$ serves as an additional measure for the 
(a)

(c)
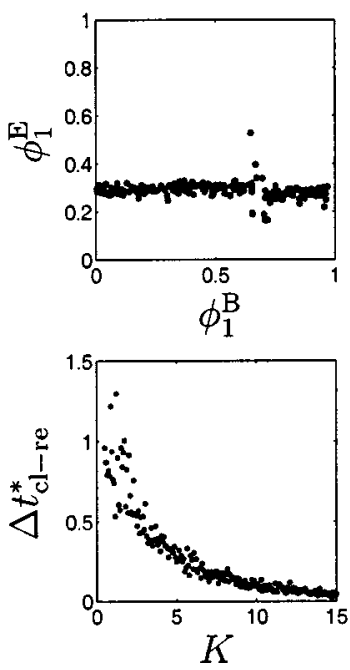

(b)

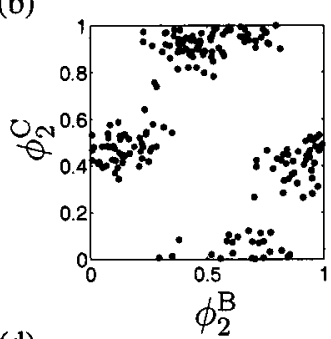

(d)

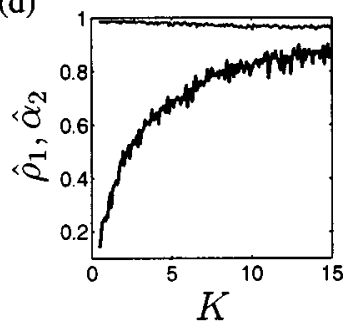

FIG. 6. Phase resetting curves [(a) and (b)]: (a) $\phi_{1}^{\mathrm{E}}$ is plotted over $\phi_{1}^{\mathrm{B}}$, (b) $\phi_{2}^{\mathrm{C}}$ is plotted over $\phi_{2}^{\mathrm{B}} ; \phi_{j}^{\mathrm{B}}=\phi_{j}(0)$ is the phase of the $j$ th oscillator at the beginning of the stimulation, $\phi_{1}^{\mathrm{E}}$ is the phase of oscillator 1 at the end of the stimulation, $\phi_{2}^{\mathrm{C}}=\phi_{2}\left(t_{\mathrm{cl}}^{(2)}\right)$ is the phase of oscillator 2 at the time when the $\mathrm{CT}$ response clustering is maximal $\left[t_{\mathrm{cl}}^{(2)}=0.56\right.$, see Fig. 5(g)]. [(c) and (d)] The coupling strength $K$ is varied between 0.5 and 15 (with all other parameters as in Fig. 5). $\Delta t_{\text {cl-re }}^{*}$ from Eq. (50), i.e., the normalized transmission time between the maximal reset of oscillator 1 and the maximal antiphase CT response clustering of oscillator 2 , is plotted in (c). $\hat{\rho}_{1}$ (thin line), the maximal value of the resetting index of oscillator 1 from Eq. (45), and $\hat{\alpha}_{2}$ (thick line), the maximal value of the antiphase clustering index of oscillator 2 from Eq. (51), are shown in (d).

$n: m$ synchronization index $\sigma_{n, m}(t)$. Large values of $\Upsilon_{n, m}(t)$ correspond to small values of $\sigma_{n, m}(t)$ and vice versa, as demonstrated by Figs. 5(i) and 5(r).

In fact, for the analysis of the presented signals, generated by numerical simulations of Eqs. (3) and (4), $\Lambda_{j}^{(\nu)}(t)$ and $\lambda_{j}^{(\nu)}(t)(\nu=1,2)$ as well as $\Upsilon_{n, m}(t)$ and $\sigma_{n, m}(t)$ provide very similar and alternative information, respectively.

\section{Comparison with the standard cross-trial analysis}

Due to the reset of oscillator 1 , the corresponding CT averaged signal $\bar{x}_{1}$ from Eq. (1) shows an oscillatory response [Fig. 7(a)], similar to that one plotted in Fig. 2(d). In contrast, as a consequence of the antiphase $\mathrm{CT}$ response clustering the CT averaged signal $\bar{x}_{2}$ of oscillator 2 vanishes [Fig. 7(c)]: The poststimulus $\bar{x}_{2}$ stays within the prestimulus range given by the first and 99th percentile of the prestimulus distribution of $\bar{x}_{2}$. Hence, with CT averaging the transmission of the stimulus' action cannot be detected.

Because of the reset of oscillator 1 , at the end of the stimulation its CT standard deviation $\varrho_{1}$ is clearly smaller compared to the prestimulus range. However, during its following reincrease, $\varrho_{1}$ undergoes an artificial oscillation with twice the frequency of oscillator 1 [Fig. 7(b)]. The same type of artificial oscillation is observed in $\varrho_{2}$, the CT standard deviation of oscillator 2 [Fig. 7(d)]. Such artificial oscillations with typically twice the frequency of the stimulus-

(a)

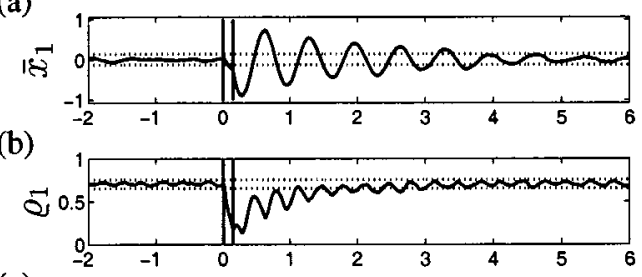

(c)

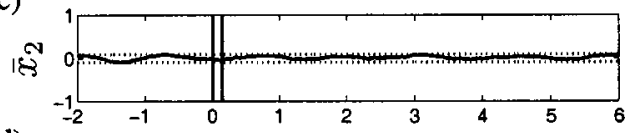

(d)

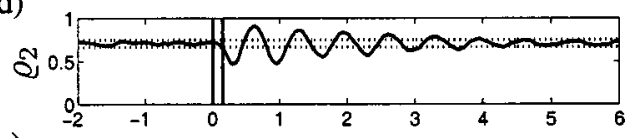

(e)

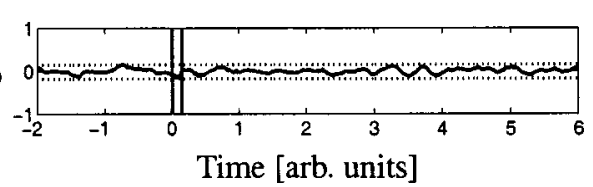

FIG. 7. CT averaged signals $\bar{x}_{1}$ (a) and $\bar{x}_{2}$ (c) from Eq. (1), CT standard deviation $\varrho_{1}$ (b) and $\varrho_{2}$ (d) from Eq. (41), and CT cross correlation $C$ (e) from Eq. (42) of the simulation shown in Fig. 5. Lower and upper dotted lines indicate the corresponding 1st and 99th percentile of the corresponding prestimulus distribution in the interval $[-8,0]$.

locked responses are the reason why the CT standard deviation is not an appropriate method for the analysis of stimulus-locked responses [30].

To detect stimulus-locked linear correlations between the two oscillators I compute the CT cross correlation $C$ from Eq. (42). There are no stimulus-locked changes of $C$; rather $C$ remains within the prestimulus range throughout the whole poststimulus period [Fig. 7(e)]. In particular, $C$ is not able to detect the stimulus-locked transient desynchronization revealed with the $1: 2$ synchronization index $\sigma_{1,2}$ from Eq. (19) [Fig. 5(j)] and the related entropy based index $\eta_{1,2}$ from Eq. (26) [Fig. 5(k)].

\section{1:3 coupling}

Depending on the coupling mechanism, one may also observe CT response clustering of higher order, i.e., there may be three or more clusters of different responses across trials. For illustration, I consider 1:3 coupled oscillators with eigenfrequencies $\omega_{1} \approx 3 \omega_{2}$ (Fig. 8). A resetting stimulus $S\left(\psi_{1}\right)$ $=I \cos \psi_{1}$ is administered to oscillator 1 [Fig. 8(a)]. The reset is detected with the resetting index $\rho_{1}$ from Eq. (20) [Fig. 8(b)] and the entropy based index $\mu_{1}$ from Eq. (25) [Fig. $8(\mathrm{c})]$. The corresponding phase resetting curve shows that oscillator 1 is reset, irrespective of the initial phase $\phi_{1}^{\mathrm{B}}$ [Fig. $8(\mathrm{~m})]$.

In contrast, the phase of oscillator 2 is not stereotypically reset to one particular value. Rather, by adapting its phase to the phase of oscillator 1, oscillator 2 may choose one of three different, equidistant stable states, as described in Sec. VI. This is shown with a phase resetting curve, where $\phi_{2}^{\mathrm{C}}$ is plotted over $\phi_{2}^{\mathrm{B}}$ [Fig. 8(n)]. $\phi_{2}^{\mathrm{C}}$ is the phase of oscillator 2 at 
(a)

(b)

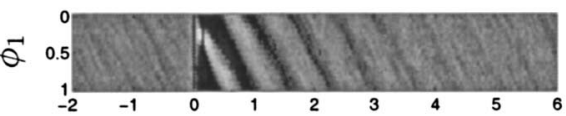

(c)

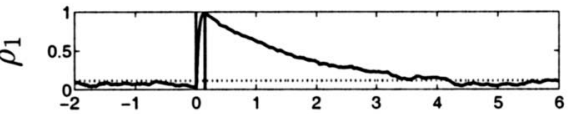

(d)

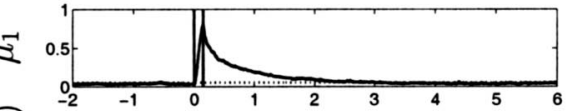

(e)

(f)

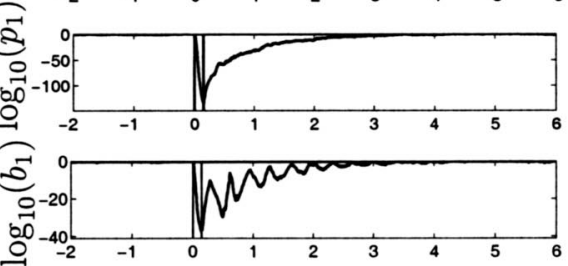

हले

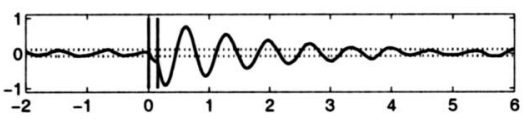

(g)

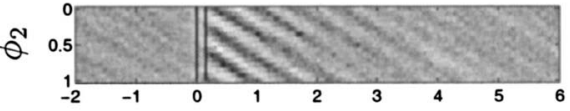

(h)

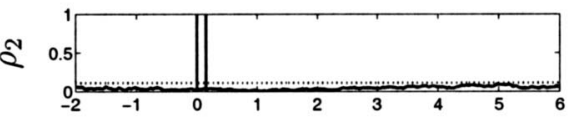

(i)

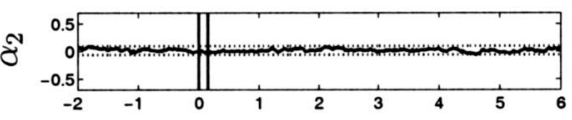

(j)

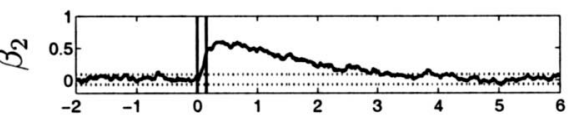

(k)

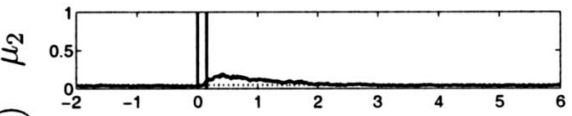

(1)

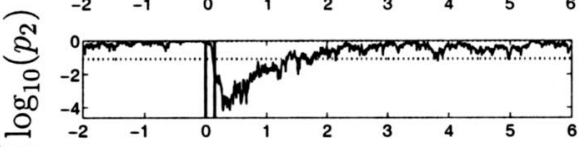

(m)

(n)

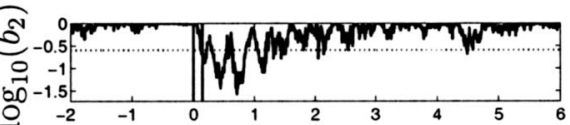

(n)

|से

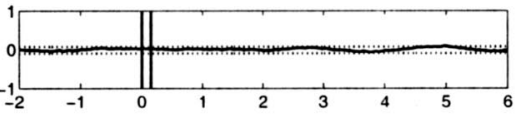

Time [arb. units]
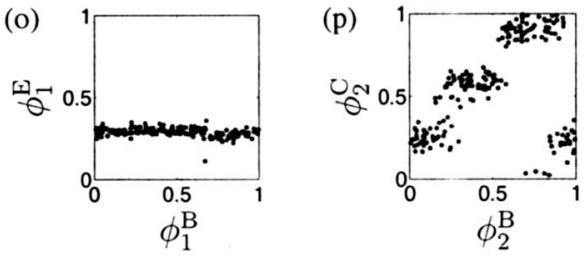

the time when the $\mathrm{CT}$ response three clustering is maximal, i.e., when the CT three-clustering index $\beta_{2}$ from Eq. (22) is maximal [at time $t_{\mathrm{cl}}^{(2)}=0.44$, Fig. $8(\mathrm{i})$ ]. $\phi_{2}^{\mathrm{B}}$ is the phase of oscillator 2 at the beginning of the stimulation. Approximately a third of a cycle $[0,1]$ of $\phi_{2}^{\mathrm{B}}$ is connected with one of three equidistant values of $\phi_{2}^{\mathrm{C}}$ (modulo 1 ), respectively.
FIG. 8. A strong stimulus $S\left(\psi_{1}\right)=I \cos \psi_{1}$ from Eq. (3) causes a reset of oscillator 1 and, by transmission, a CT response three clustering of oscillator 2. CT distributions from Eq. (17): $\left\{\phi_{1}(t\right.$ $\left.\left.+\tau_{k}\right)\right\}_{k=1, \ldots, l}$ in (a) and $\left\{\phi_{2}\left(t+\tau_{k}\right)\right\}_{k=1, \ldots, l}$ in (g) ( 0 is black and maximal values are white). Indices from Eqs. (20)-(25): $\rho_{1}$ in (b), $\mu_{1}$ in (c), $\rho_{2}$ in (h), $\alpha_{2}$ in (i), $\beta_{2}$ in (j), and $\mu_{2}$ in (k). CT averaged signals from Eq. (1): $\bar{x}_{1}$ in (f) and $\bar{x}_{2}$ in (n). Base 10 logarithm of the $P$ values of the CT Kuiper test for randomness: $\log _{10}\left(p_{j}\right)$ from Eq. (39) for the CT distribution of the phase of oscillator $j$ in (d) and (1). Base 10 logarithm of the $P$ values of the CT KolmogorovSmirnov test for randomness: $\log _{10}\left(b_{j}\right)$ from Eq. (35) for the CT distribution of the phase of oscillator $j$ in (e) and (m). Same format as in Fig. 2. Prestimulus time window used for calculating the significance levels: $\left[t_{a}, 0[=[-8,0[\right.$. Parameters of Eqs. (3) and (4): $K=3.5, n=1, m=3, \omega_{1} /(2 \pi)=1.5, \omega_{2} /(2 \pi)=0.498, D=1, I=40$, $\theta=0, t_{\text {win }}=16$ [see Eq. (16)], stimulus duration $=0.15$, and number of stimuli $l=200$. Results are stable with respect to variations of $l$ between 50 and 2000 and more. Phase resetting curves [(o) and (p)]: (o) $\phi_{1}^{\mathrm{E}}$ is plotted over $\phi_{1}^{\mathrm{B}}$, (p) $\phi_{2}^{\mathrm{C}}$ is plotted over $\phi_{2}^{\mathrm{B}} ; \phi_{j}^{\mathrm{B}}$ $=\phi_{j}(0)$ is the phase of the $j$ th oscillator at the beginning of the stimulation, $\phi_{1}^{E}$ is the phase of oscillator 1 at the end of the stimulation, $\phi_{2}^{\mathrm{C}}=\phi_{2}\left(t_{\mathrm{cl}}^{(2)}\right)$ is the phase of oscillator 2 at the time when the CT response three clustering is maximal $\left[t_{\mathrm{cl}}^{(2)}=0.44\right.$, see $\left.(\mathrm{j})\right]$.

The CT response three clustering is detected with the CT three-clustering index $\beta_{2}$ from Eq. (22) [Fig. 8(i)] and, less pronounced, also with the entropy based index $\mu_{2}$ [Fig. 8(j)], whereas it cannot be detected with the resetting index $\rho_{2}$ [Fig. 8(g)] or the CT antiphase response clustering index $\alpha_{2}$ from Eq. (21) [Fig. 5(h)]. 
While the reset is connected with a nonvanishing averaged response $\bar{x}_{1}$ from Eq. (1) [Fig. 8(e)], the CT averaged signal $\bar{x}_{2}$ of oscillator 2 is averaged out [Fig. 8(1)], because the three clusters of responses are of similar size and have equidistant phases [Figs. 8(f) and 8(n)].

\section{E. Cross-trial Kuiper test}

The strong reset of oscillator 1 is detected by the indices $\rho_{1}$ and $\mu_{1}$ as well as with the CT Kuiper test: The resetting index $\rho_{1}$ from Eq. (20) and the entropy based index $\mu_{1}$ from Eq. (25) increase beyond their 99th prestimulus percentile [Figs. 8(b) and 8(c)], respectively. The $P$ value $p_{1}$ from Eq. (35), obtained with the CT Kuiper test, decreases below its first prestimulus percentile [Fig. 8(d)]. The duration of the reset, as given by the pattern of the CT distribution of $\phi_{1}$, is best represented by the time course of $\rho_{1}$ and $\log _{10}\left(p_{1}\right)$.

The CT response three clustering is specifically detected with the CT three-clustering index $\beta_{2}$ from Eq. (22) [Fig. $8(\mathrm{j})$ ]. Furthermore, it shows up as an increase of the entropy based index $\mu_{1}$ beyond its 99th prestimulus percentile [Fig. 8(j)]. The $P$ value $p_{2}$ of the CT Kuiper test, decreases below its first prestimulus percentile [Fig. 8(1)]. This means that during the $\mathrm{CT}$ response three clustering the $\mathrm{CT}$ distribution of $\phi_{2}$ is significantly different from the prestimulus uniform distribution. The duration of the CT response three clustering, i.e., the pattern of the CT distribution of $\phi_{2}$, is best reflected by the time course of $\beta_{2}$. Note, the CT Kuiper test detects both a reset and a CT response clustering as epochs during which the CT distribution of the phase differs significantly from a uniform distribution. With the CT Kuiper test one cannot distinguish between a reset and a CT response clustering.

The Kuiper test is the circular version of the KolmogorovSmirnov test (see Secs. III D and III E). Unlike the CT Kuiper-test, the CT Kolmogorov-Smirnov test is no appropriate method for detecting stimulus-locked dynamics. The $P$ value from Eq. (35) obtained with the CT KolmogorovSmirnov test displays artificial oscillations that are not related to the stimulus-locked process under consideration [Figs. 8(e) and 8(m)]. The origin of these oscillations is explained in Sec. X. In particular, due to these oscillations the epoch with CT response three clustering is not detected as a whole [Fig. 8(m)]. Rather, during this epoch $\log _{10}\left(b_{2}\right)$ from Eq. (35), the base 10 logarithm of the $P$ value of the CT Kolmogorov-Smirnov test, oscillates around its first prestimulus percentile.

\section{NO DIRECT CT AVERAGED RESPONSE, BUT TRANSMISSION OF THE CT AVERAGED RESPONSE}

In biological systems the response to a stimulus often depends on the phase of the oscillation at which the stimulus is administered [3,5]. For instance, in spinal cord physiology there is the so-called reflex reversal, which means that depending on the initial phase, a network of oscillatory neurons may generate antiphase responses, i.e., either an excitation of the flexor muscle or an excitation of the extensor muscle
[52]. The reflex reversal is essential for an adaptive control of locomotion.

Let me consider a simple stimulation mechanism which causes an antiphase CT response clustering of the stimulated oscillator in a way comparable to the reflex reversal. For this, I use $S\left(\psi_{1}\right)=I \cos \left(2 \psi_{1}\right)$. Depending on the initial phase, oscillator 1 generates one of two antiphase responses. This is shown by means of the phase resetting curve, where $\phi_{1}^{\mathrm{E}}$ plotted over $\phi_{1}^{\mathrm{B}}$ for all $l$ trials [Fig. $\left.9(\mathrm{k})\right]$. $\phi_{1}^{\mathrm{B}}$ and $\phi_{1}^{\mathrm{E}}$ denote the phase of oscillator 1 at the beginning and at the end of the stimulation, respectively. $\phi_{1}^{\mathrm{E}}$ is close to 0.66 for $0.32<\phi_{1}^{\mathrm{B}}<0.84$, whereas $\phi_{1}^{\mathrm{E}}$ is close to 0.16 else, with little overlap between the two regions of $\phi_{1}^{\mathrm{B}}$ [Fig. 9(k)]. Accordingly, the CT distribution of the phase of oscillator 1 shows two pronounced antiphase peaks at the end of the stimulation [Fig. 9(a)]. This, in turn, corresponds to high values of $\alpha_{1}$, the antiphase CT clustering index from Eq. (21) [Fig. 9(b)]. Consistently, the clustering is not detected with the resetting index $\rho_{1}$ from Eq. (20). Noise makes the clustering fade away in the course of the poststimulus transient.

As explained in Sec. VI, as a consequence of the $1: 2$ coupling, in the stable synchronized state the same value of $\phi_{2}$ belongs to the two antiphase values of $\phi_{1}$. Correspondingly, after the stimulation one peak of the CT distribution of $\phi_{2}$ is formed [Fig. 9(e)]. However, the phase resetting curve shows that the reset caused by transmission is less pronounced [Fig. 9(1)] compared to a reset caused by direct and strong stimulation [Fig. 6(a)]. Nevertheless, the reset is detected with the resetting index $\rho_{2}$ from Eq. (20) [Fig. 9(f)], whereas the antiphase CT clustering index $\alpha_{2}$ from Eq. (21) falls below the prestimulus range. An increase of $\rho_{2}$ combined with a decrease of $\alpha_{2}$ is indicative of a reset [compare Figs. 5(b) and 5(c)]. The reset causes a transient desynchronization that vanishes as soon as the phase of oscillator 2 is adapted to the phase of oscillator 1 [Figs. 9(i) and 9(j)].

Due to the antiphase CT clustering, the averaged signal $\bar{x}_{1}$ of oscillator 1 vanishes [Fig. 9(d)], whereas the reset of oscillator 2 is connected with a nonvanishing averaged response of $\bar{x}_{2}$ [Fig. 9(h)].

\section{NEITHER DIRECT CT AVERAGED RESPONSE NOR TRANSMISSION OF THE CT AVERAGED RESPONSE}

The same stimulus $S\left(\psi_{1}\right)=I \cos \left(2 \psi_{1}\right)$ is administered to oscillator 1 as in Sec. VIII. This causes an antiphase CT response clustering [Fig. 10(a)], which is detected with the antiphase CT clustering index $\alpha_{1}$ from Eq. (21) [Fig. 10(c)], but not with the resetting index $\rho_{1}$ from Eq. (20) [Fig. 10(b)]. Unlike in Sec. VIII, the two oscillators are 1:1 coupled, so that there is only one stable phase relation between the two oscillators. Hence, the antiphase CT response clustering is transmitted onto oscillator 2 [Figs. 10(e) and 10(g)]. Because of the antiphase CT response clustering the averaged signals $\bar{x}_{j}$ from Eq. (1) of both oscillators vanish.

There are numerous other suitable combinations of coupling mechanism and stimulation mechanism for which both oscillators have no averaged response. For instance, in case of a 2:3 coupling, administration of a stimulus $S\left(\psi_{1}\right)$ 
(a)

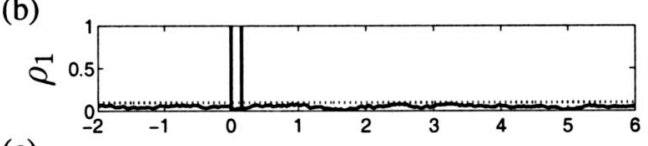

(c)
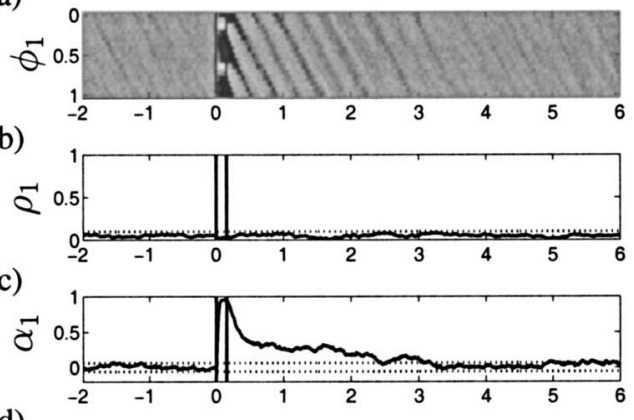

(d)

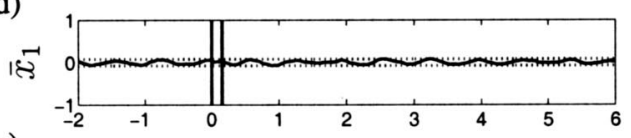

(e)

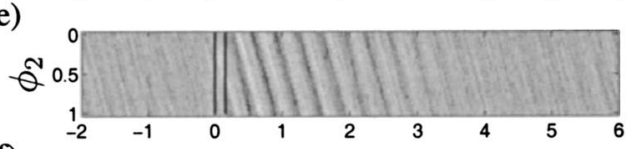

(f)

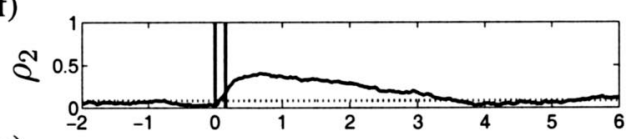

(g)

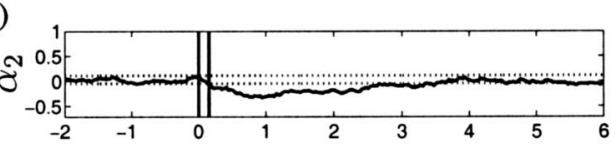

(h)

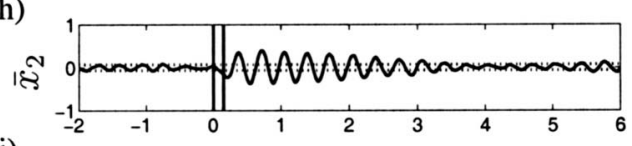

(i)

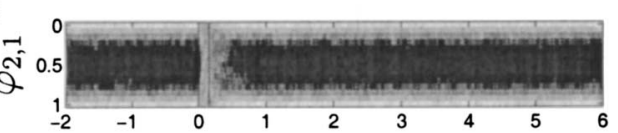

(j)
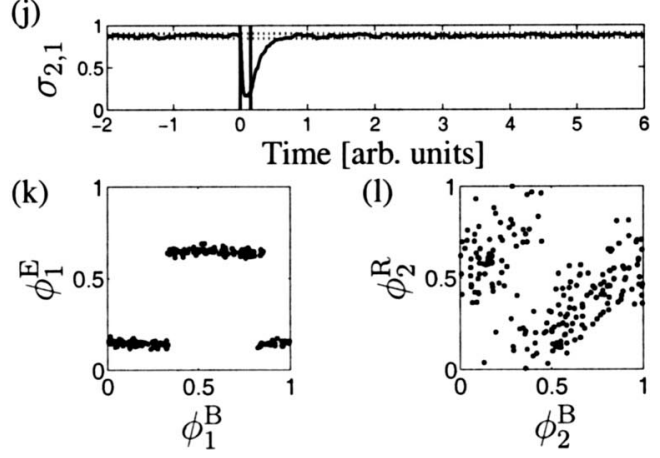

$=I \cos \left(2 \psi_{1}\right)$ causes an antiphase CT response clustering, which via transmission shows up as CT response three clustering.

\section{COMPARISON OF THE CT DATA ANALYSIS TECHNIQUES WITH SYNTHETIC RESPONSES}

To illustrate and summarize important features of the data analysis techniques from Secs. III and IV, they are applied to noise-free, idealized responses. These artificial responses are not generated by the model given by Eqs. (3) and (4), but simply defined in order to reveal advantages and drawbacks of the different data analysis methods. I consider an ensemble of responses of both oscillators given by
FIG. 9. A strong stimulus $S\left(\psi_{1}\right)=I \cos \left(2 \psi_{1}\right)$ from Eq. (3) causes an antiphase CT response clustering of oscillator 1 , which by transmission via the 2:1 coupling leads to a reset of oscillator 2. CT distributions from Eq. (17): $\left\{\phi_{1}\left(t+\tau_{k}\right)\right\}_{k=1, \ldots, l}$ in (a), $\left\{\phi_{2}(t\right.$ $\left.\left.+\tau_{k}\right)\right\}_{k=1, \ldots, l}$ in (e), and $\left\{\varphi_{2,1}\left(t+\tau_{k}\right)\right\}_{k=1, \ldots, l}$ in (h) $(0$ is black and maximal values are white). Indices from Eqs. (19)-(21): $\rho_{1}$ in (b), $\alpha_{1}$ in (c), $\rho_{2}$ in (f), and $\sigma_{2,1}$ in (j). CT averaged signals from Eq. (1): $\bar{x}_{1}$ in (d) and $\bar{x}_{2}$ in (h). Same format as in Fig. 2. Prestimulus time window used for calculating the significance levels: $\left[t_{a}, 0[=[\right.$ $-8,0[$. Parameters of Eqs. (3) and (4): $K=3.5, n=2, m=1$, $\omega_{1} /(2 \pi)=1.5, \omega_{2} /(2 \pi)=2.99, D=1, I=40, \theta=0, t_{\text {win }}=16$ [see Eq. (16)], stimulus duration $=0.15$, and number of stimuli $l=200$. Results are stable with respect to variations of $l$ between 50 and 2000 and more. Phase resetting curves $[(\mathrm{j})$ and $(\mathrm{k})]$ : $(\mathrm{j}) \phi_{1}^{\mathrm{E}}$ is plotted over $\phi_{1}^{\mathrm{B}}$, (k) $\phi_{2}^{\mathrm{R}}$ is plotted over $\phi_{2}^{\mathrm{B}} ; \phi_{j}^{\mathrm{B}}=\phi_{j}(0)$ is the phase of the $j$ th oscillator at the beginning of the stimulation, $\phi_{1}^{\mathrm{E}}$ is the phase of oscillator 1 at the end of the stimulation, $\phi_{2}^{\mathrm{R}}=\phi_{2}\left(t_{r}^{(2)}\right)$ is the phase of oscillator 2 at a time during its reset $\left[t_{\mathrm{cl}}^{(2)}=0.87\right.$, see (f)].

$$
\begin{gathered}
\phi_{1}\left(t+\tau_{k}\right)=\left[t+\varepsilon \xi_{1, k}\right] \bmod 1, \\
\phi_{2}\left(t+\tau_{k}\right)=\left[t+\Delta \phi+\varepsilon \xi_{2, k}\right] \bmod 1,
\end{gathered}
$$

for $k=1, \ldots, l$, where $\Delta \phi$ is the mean phase difference between the responses of the two oscillators, and $\left\{\xi_{j, k}\right\}_{k=1}^{l}$ is constant and normally distributed with variance 1 for $j$ $=1,2$. By varying $\varepsilon$ I modify the variance of the normal distributions of the responses. While the time $t$ increases from 0 to 1 , the CT distribution of the phase of each oscillator, $\left\{\phi_{j}\left(t+\tau_{k}\right)\right\}_{k=1, \ldots, l}$ from Eq. (17), is shifted through one cycle [Figs. 11(a) and 11(b)]. $\Delta \phi$ is chosen to be equal to 0.25 , where the features of the CT analysis methods discussed below are independent of the choice of $\Delta \phi$. 
(a)

(b)
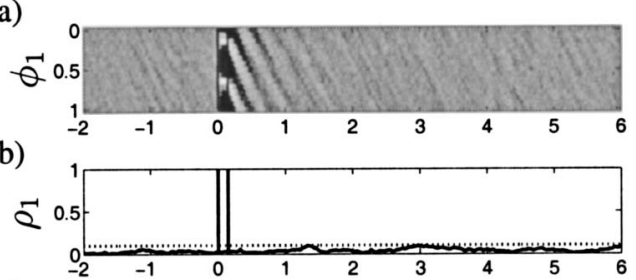

(c)

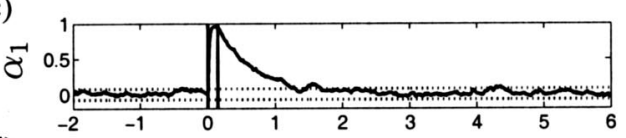

(d)

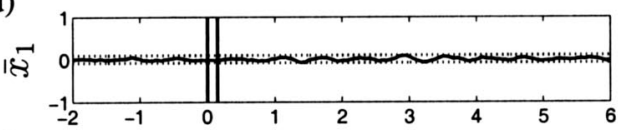

(e)

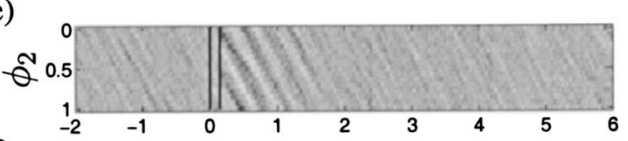

(f)

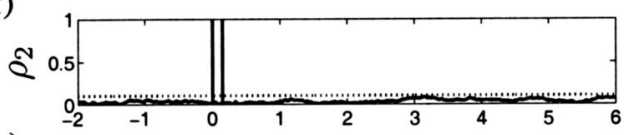

(g)

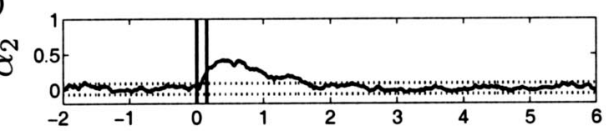

(h)

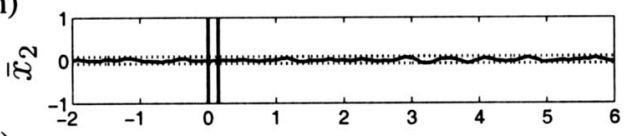

(i)

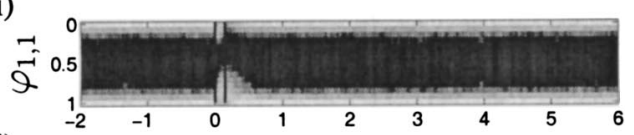

(j)

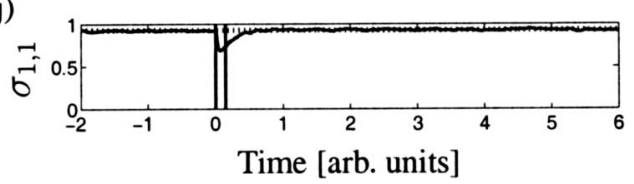

FIG. 10. A strong stimulus $S\left(\psi_{1}\right)=I \cos \left(2 \psi_{1}\right)$ from Eq. (3) causes an antiphase CT response clustering of oscillator 1 , which by transmission via the $1: 1$ coupling causes an antiphase CT response clustering of oscillator 2. CT distributions from Eq. (17): $\left\{\phi_{1}(t\right.$ $\left.\left.+\tau_{k}\right)\right\}_{k=1, \ldots, l}$ in (a), $\left\{\phi_{2}\left(t+\tau_{k}\right)\right\}_{k=1, \ldots, l}$ in (e), and $\left\{\varphi_{1,1}\left(t+\tau_{k}\right)\right\}_{k=1, \ldots, l}$ in (i) ( 0 is black and maximal values are white). Indices from Eqs. (19)-(21): $\rho_{1}$ in (b), $\alpha_{1}$ in (c), $\rho_{2}$ in (f), $\alpha_{2}$ in (g), and $\sigma_{1,1}$ in (j). CT averaged signals from Eq. (1): $\bar{x}_{1}$ in (d) and $\bar{x}_{2}$ in (h). Same format as in Fig. 2. Prestimulus time window used for calculating the significance levels: $\left[t_{a}, 0[=[-8,0[\right.$. Parameters of Eqs. (3) and (4): $K$ $=3.5, n=1, m=1, \omega_{1} /(2 \pi)=1.5, \omega_{2} /(2 \pi)=1.49, D=1, I=40, \theta=0$, $t_{\text {win }}=16$ [see Eq. (16)], stimulus duration $=0.15$, and number of stimuli $l=200$. Results are stable with respect to variations of $l$ between 50 and 2000 and more.

Note, for each oscillator the CT standard deviation (modulo 1) of the phases $\phi_{j}\left(t+\tau_{k}\right)$ of all responses $k$ $=1, \ldots, l$ is constant in time [Figs. 11(a) and 11(b)]. Furthermore, the phase difference $\varphi_{1,1}\left(t+\tau_{k}\right)$ of all responses $k$ $=1, \ldots, l$ is constant in time $t$ [Fig. 11(c)]. Accordingly, quantities measuring the extent of the stimulus locking of the responses of each single oscillator as well as their interdependence have to be constant, too.

Univariate measures. According to their definition, the resetting indices $\rho_{1}$ and $\rho_{2}$ from Eq. (20) are invariant with respect to phase shifts of the CT distribution $\left\{\phi_{j}(t\right.$ $\left.\left.+\tau_{k}\right)\right\}_{k=1, \ldots, l}$ and, thus, constant for all times $t \in[0,1]$ [Figs. $11(\mathrm{~d})$ and $11(\mathrm{e})]$.

In contrast, the entropy based stimulus locking indices $\mu_{1}$ and $\mu_{2}$ from Eq. (25) display oscillations that are due to the binning of the CT distributions. The binning is necessary for the calculation of the entropy. However, such oscillations occur predominantly for sharp CT distributions (i.e., for $\varepsilon$ $=0.001$, blue line) [Figs. 11(g) and 11(h)].

The $P$ values of the CT Kolmogorov-Smirnov test display an artificial oscillation with period 1, i.e., with the same period as the oscillation under study [Figs. 11(j) and 11(k)]. This oscillation is strongest for sharp CT distributions $(\varepsilon$ $=0.001$, blue line), but it is also present for smooth CT distributions ( $\varepsilon=1$, green line). The oscillation of the $P$ value of the CT Kolmogorov-Smirnov test is caused by an oscillation of $D$, the maximum value of the absolute difference between the two cumulative distribution functions from Eq. (32) (not shown due to space constraints). By the way, the artificial oscillations of the $P$ values of the CT Kolmogorov-Smirnov test are also observed when applying this method to signals generated by the model from Eqs. (3) and (4) [Figs. 8(e) and $8(\mathrm{~m})$ and discussions in Sec. VII E].

In contrast, the $P$ values of the CT Kuiper test are invariant with respect to a phase shift of the CT distribution of the phase. Consequently, the $P$ values of the CT Kuiper test are constant for all times $t \in[0,1]$ [Figs. $11(\mathrm{~m})$ and 11(n)]. Consequently, also when applied to numerical signals stemming from Eqs. (3) and (4), the CT Kuiper test produces no artificial oscillations [Figs. 8(d) and 8(1)].

By definition, the CT averaged signals $\bar{x}_{1}$ and $\bar{x}_{2}$ from Eq. (1) oscillate and run through one period while the CT distribution of the phase is shifted through one period [Figs. 11(p) and $11(\mathrm{q})]$.

The CT standard deviation $\varrho_{j}$ from Eq. (41) is (nearly) constant in time only for nearly vanishing variance of the responses, i.e., for $\varepsilon$ close to 0 [Figs. 11(s) and 11(t), blue lines]. On the contrary, for larger values of $\varepsilon$ one observes an artificial oscillation of $\varrho_{j}$ with a period of $T / 2$, where $T=1$ is the period of the synthetic oscillators. This oscillation occurs independently of the choice of $\Delta \phi$ from Eq. (53). The CT standard deviation $\varrho_{j}$ attains its greatest values at times when the corresponding $\mathrm{CT}$ averaged signal $\bar{x}_{j}$ is close to zero crossings. The artificial oscillations of $\varrho_{j}$ are also observed in numerical simulations of Eqs. (3) and (4) [Figs. 7(b) and 7(d)].

Bivariate measures. As already mentioned, the phase difference $\varphi_{1,1}\left(t+\tau_{k}\right)$ of all responses $k=1, \ldots, l$ remains constant in time $t$. Consequently, $\left\{\varphi_{1,1}\left(t+\tau_{k}\right)\right\}_{k=1, \ldots, l}$, the CT distribution of the 1:1 phase difference from Eq. (17), is constant in time, too. Therefore, the $1: 1$ synchronization index $\sigma_{1,1}$ from Eq. (19) [Fig. 11(f)], the entropy based 1:1 synchronization index $\eta_{1,1}$ from Eq. (26) [Fig. 11(i)], the $P$ value from Eq. (36) of the CT Kolmogorov-Smirnov test for the CT distribution of the 1:1 phase difference [Fig. 11(1)], 

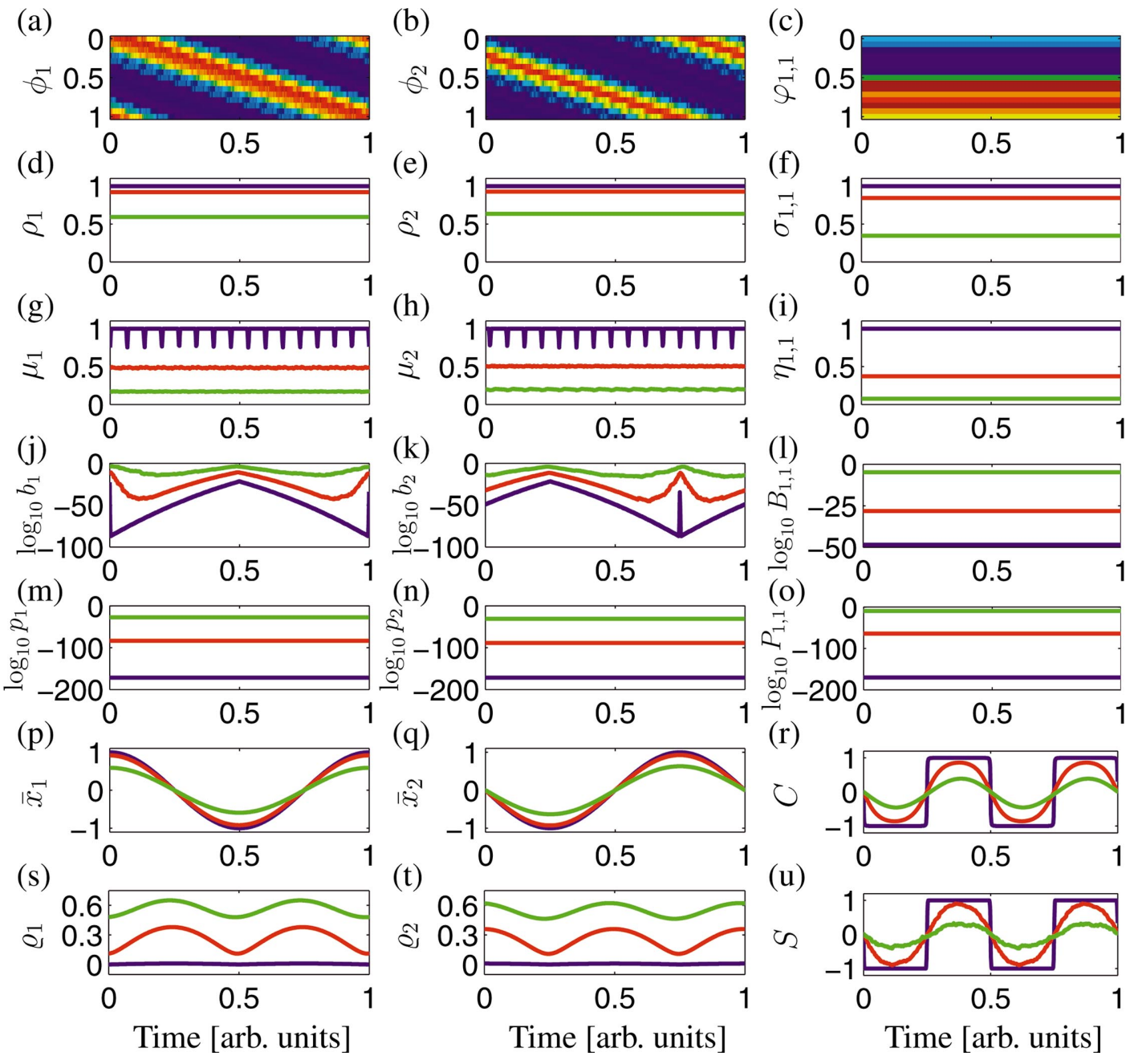

FIG. 11. (Color) To demonstrate the phase dependence of particular CT data analysis techniques, the CT methods from Secs. III and IV are applied to noise-free, idealized responses, defined by Eqs. (52) and (53). These artificial responses are not generated by the model from Eqs. (3) and (4). Rather, by definition they are given by $\phi_{1}\left(t+\tau_{k}\right)=\left[t+\varepsilon \xi_{1, k}\right] \bmod 1$ and $\phi_{2}\left(t+\tau_{k}\right)=\left[t+\Delta \phi+\varepsilon \xi_{2, k}\right] \bmod 1$ with $\Delta \phi=0.25$ for $k=1, \ldots, l$, where $\left\{\xi_{j, k}\right\}_{k=1}^{l}$ is constant and normally distributed with variance 1 , and $\varepsilon=0.01$ (blue line), 0.1 (red line), 1 (green line). Note, the period of both $\phi_{1}$ and $\phi_{2}$ equals 1 . CT distributions from Eq. (17) for $\varepsilon=0.1$ are shown as time-dependent histograms of $\phi_{j}$ and $\varphi_{1,1}$ calculated across trials for each time $t$ relative to stimulus onset within the time window $\left[t_{a}, t_{b}\right]:\left\{\phi_{1}\left(t+\tau_{k}\right)\right\}_{k=1, \ldots, l}$ in $(\mathrm{a}),\left\{\phi_{2}\left(t+\tau_{k}\right)\right\}_{k=1, \ldots, l}$ in (b), and $\left\{\varphi_{1,1}\left(t+\tau_{k}\right)\right\}_{k=1, \ldots l}$ in (c) [color scale ranges from dark blue (zero), blue, light blue, green, yellow, orange, red to crimson (maximal values)]. Resetting indices $\rho_{1}$ and $\rho_{2}$ from Eq. (20) in (d) and (e); $n: m$ synchronization index $\sigma_{1,1}$ from Eq. (19) in (f); entropy based stimulus locking indices $\mu_{1}$ and $\mu_{2}$ from Eq. (25) in (g) and (h); entropy based $n: m$ synchronization index $\eta_{1,1}$ from Eq. (26) in (i); base 10 logarithm of the $P$ values of the CT Kolmogorov-Smirnov test for randomness: $\log _{10}\left(b_{1}\right)$ and $\log _{10}\left(b_{2}\right)$ from Eq. (35) for the CT distribution of the phases in (j) and (k); $\log _{10}\left(B_{n, m}\right)$ from Eq. (36) for the CT distribution of the $n: m$ phase difference in (1); base 10 logarithm of the $P$ values of the CT Kuiper test for randomness: $\log _{10}\left(p_{1}\right)$ and $\log _{10}\left(p_{2}\right)$ from Eq. (39) for the CT distribution of the phases in $(\mathrm{m})$ and $(\mathrm{n})$; $\log _{10}\left(P_{n, m}\right)$ from Eq. (40) for the CT distribution of the $n: m$ phase difference in (o); CT averaged signals $\bar{x}_{1}$ and $\bar{x}_{2}$ from Eq. (1) in (p) and (q), CT cross correlation $C$ from Eq. (42) in (r), CT standard deviations $\varrho_{1}$ and $\varrho_{2}$ from Eq. (41) in (s) and (t), and CT sign cross correlation $S$ from Eq. (43) in (u).

and the $P$ value from Eq. (40) of the CT Kuiper test for the CT distribution of the $1: 1$ phase difference [Fig. 11(o)] are constant for all times $t \in[0,1]$. Note, for time varying CT distributions of the $1: 1$ phase difference $\eta_{1,1}$ and the $P$ value of the CT Kolmogorov-Smirnov test for the CT distribution of the 1:1 phase difference produce the same artificial oscillations as in the case of the univariate analysis explained above.

Although the CT distribution of the 1:1 phase difference remains constant in time, the CTCC, $C$ from Eq. (42) [Fig. 11(r)], and the CTSCC, $S$ from Eq. (43) [Fig. 11(u)], of these synthetic stimulus-locked responses "artificially" oscillate with increasing time $t$, i.e., with increasing phases $\phi_{j}$ although the phase difference $\varphi_{n, m}$ remains constant. These oscillations occur for all values of the phase difference $\Delta \phi$. For $\varepsilon=0$ [blue lines in Figs. 11(r) and 11(u)], $C$ and $S$ are $=1$ or $=-1$ if the signals $x_{1}=\cos \left(2 \pi \phi_{1}\right)$ and $x_{2}$ have the same or different sign, respectively. $C$ and $S$ vanish when $x_{1}$ and $x_{2}$ have zero crossings. A constant gitter of the phases $[\varepsilon>0$, red and green lines in Figs. 11(r) and 11(u)] smoothens the 
changes of $C$ and $S$ around the zero crossings, so that oscillations with twice the oscillators' frequency occur-although the phase difference $\varphi_{n, m}$ of all pairs of responses is constant. CTSCC and CTCC nearly coincide. Applied to signals stemming from simulations of the model given by Eqs. (3) and (4), the time courses of both CTCC and CTSCC are practically the same (not shown due to space constraints). In particular, CTSCC is not superior to CTCC with respect to the artificial oscillations that are not related to stimulus-locked synchronization.

\section{DISCUSSION}

In this paper, I have presented a model which allows one to study basic transmission properties of stimulus-locked responses in two coupled phase oscillators, where only one oscillator is stimulated. Furthermore, I have explained how to detect such dynamics reliably with data analysis techniques based on stochastic phase resetting (Sec. III). The major results are the following.

(a) In 1:1 coupled phase oscillators the transmission time of the CT averaged responses, i.e., the difference in time between the maxima of the CT averaged responses of both oscillators, directly corresponds to the phase difference in the stable synchronized state given by Eq. (13) with integer multiples of the oscillators' mean period added to it, where the integer value of added periods depends on the coupling strength (Fig. 3, Sec. V). Thus, the transmission time of the averaged responses primarily corresponds to features of the coupling mechanism and the detuning. In particular, the transmission time of the CT averaged responses is not a quantity that reflects the time elapsing due to the stimulus' action being transmitted between the two oscillators. This contradicts the assumption used in the evoked response literature $[6,10,11,26]$. In contrast, with the stochastic phase resetting analysis from Sec. III [see Eq. (44)] the transmission time of the stimulus-locked responses is reliably assessed by detecting the time passing by between the maximal resets of both oscillators. In general, for $n: m$ coupling the transmission time is determined by detecting the time elapsing between the maximal response events of both oscillators, e.g., the maximal reset of oscillator 1 and the maximal CT response clustering of oscillator 2 [see Eq. (50) in Sec. VII A].

(b) $n: m$ coupling is common in the nervous system. For instance, there are several interacting brain rhythms such as $\alpha$ rhythm (around $10 \mathrm{~Hz}$ ) and $\beta$ rhythm (around $20 \mathrm{~Hz}$ ), which have an $n: m$ relationship of their dominant frequencies, where $n$ and $m$ are small integers [6,26]. In the model given by Eqs. (3) and (4), $n: m$ coupling (with $n \neq 1$ and/or $m \neq 1$ and $n \neq m$ ) typically leads to a bistability or multistability of stable synchronized states of the two-phase oscillators (modulo $2 \pi$ ), although a simple in-phase synchronizing coupling of lowest order is used (see Secs. II B and VI). For this reason, after stimulation the two oscillators may approach qualitatively different stable states across trials. Consequently, in two $n: m$ coupled phase oscillators either one or both oscillators may generate an antiphase $\mathrm{CT}$ response clustering, which cannot be detected with the $\mathrm{CT}$ averaging from
Eq. (1). Correspondingly, with respect to the standard averaged response, there are three different cases, where CT averaging fails.

(i) Only the directly stimulated oscillator shows an averaged response, and there is no transmission of the averaged response (Sec. VII). Interestingly, the antiphase CT response clustering is not only detected with the stochastic phase resetting analysis (Fig. 5), but this data analysis approach reveals striking similarities between qualitatively different transmission processes: The normalized transmission time between the reset of oscillator 1 and the CT response clustering of oscillator 2 from Eq. (50) depends on $K$ in a similar way as the normalized transmission time of the reset from Eq. (44) in case of a transmitted reset (compare Figs. 3 and $6)$.

(ii) The averaged response is not observed in the directly stimulated oscillator, but in the oscillator coupled to it (Sec. VIII).

(iii) Neither the directly stimulated nor the other oscillator displays an averaged response (Sec. IX).

For the study of the transmission time, the coupling strength was restricted to values up to 15 (Figs. 3 and 6). Given the model parameters used in Figs. 3 and 6, values of $K$ greater than 15 lead to a very strong synchronization connected with a sharply peaked CT distribution $\left\{\varphi_{n, m}(t\right.$ $\left.\left.+\tau_{k}\right)\right\}_{k=1, \ldots, l}$. Sharp distributions of this kind are typically not observed in biological data, neither under healthy nor under pathological situations $[53,54]$.

The coupling in Eqs. (3) and (4) is symmetrical. All of the dynamical phenomena presented in this paper occur also in the case of nonidentical coupling strength. Obviously, the only necessary condition is that the oscillator, which is not stimulated, is coupled to the oscillator, which is stimulated. Otherwise there is no way for the stimulus' effect to be transmitted.

By the same token, also for nonsymmetric phase shifts $\theta_{1} \neq \theta_{2}$ instead of $\theta$ in the sine coupling terms of Eqs. (3) and (4), one observes the same dynamical phenomena as presented in this paper. Also, the data analysis from Sec. III works for all types of nonsymmetric phase shifts $\theta_{1} \neq \theta_{2}$ as in the symmetric case. However, there is one special case occurring for symmetric coupling [as in Eqs. (3) and (4)] with $\theta_{1}-\theta_{2}=\pi$ modulo $2 \pi$. In this case the model equation reads

$$
\begin{gathered}
\dot{\psi}_{1}=\omega_{1}-K \sin \left(n \psi_{1}-m \psi_{2}+\theta_{1}\right)+X(t) S\left(\psi_{1}\right)+F_{1}(t), \\
\dot{\psi}_{2}=\omega_{2}-K \sin \left(m \psi_{2}-n \psi_{1}-\theta_{2}\right)+F_{2}(t) .
\end{gathered}
$$

Both couplings have opposite tendencies which, due to the symmetric coupling strength, remain balanced: No synchronization occurs under spontaneous conditions, i.e., for $X=0$. Furthermore, for nonvanishing detuning $n \omega_{1}-m \omega_{2}$ the instantaneous frequencies of both oscillators, $\dot{\psi}_{1}$ and $\dot{\psi}_{2}$, undergo oscillations. Hence, for nonvanishing detuning the interaction between the two oscillators leads to a frequency modulation instead of a phase synchronization. In the sim- 
plest case, for $n=m=1$, pulsatile stimulation of oscillator 1 (as studied in Sec. V) leads to a reset which by transmission causes a reset of oscillator 2. In general, there is transmission of the stimulus' action without synchronization. Since this phenomenon occurs only for particular parameter values (symmetric coupling and $\theta_{1}-\theta_{2}=\pi$ modulo $2 \pi$ ), a detailed description will be presented elsewhere, in order not to overload the present paper.

Note, there is a fundamental difference between the concept of stimulus-locked synchronization used in this study on the one hand and the concept of stationary or quasistationary stochastic phase synchronization on the other hand. Stochastic phase synchronization refers to processes which are not transient, but evolve on a long time scale, i.e., for time $t$ $\rightarrow \infty$. The typical scenario of stochastic phase synchronization is given by two self-sustained oscillators without any pulsatile stimulation. The basic feature of stochastic phase synchronization is that two oscillators are able to maintain a stable phase relationship during a long period of time, although they are subject to random forces. Accordingly, stochastic phase synchronization was defined as appearance of one or more prominent peaks in the distribution of the phase difference during a sufficiently long observation $[55,56]$.

In contrast, the analysis of stimulus-locked dynamics of the phase difference (Sec. III) is by no means restricted to the detection of stimulus-locked phase synchronized states (i.e., states, with rather constant $n: m$ phase difference in time plus possible $2 \pi$ jumps), which are time locked to the stimulus. Rather, the $n: m$ phase difference $\varphi_{n, m}$ may also undergo stimulus-locked transients during which $\varphi_{n, m}$ varies in time. For instance, during the prestimulus epoch there may be an in-phase synchronization. The stimulus causes an antiphase synchronization, which via a branching (two clustering) comes back to an in-phase synchronization again [28-30].

Using formulas from circular statistics [33], in Sec. III the CT mean angular deviation $\Lambda_{j}^{(1)}(t)$ for a unimodal CT distribution $\left\{\phi_{j}\left(t+\tau_{k}\right)\right\}_{k=1, \ldots, l}$ and the CT mean angular deviation $\Lambda_{j}^{(2)}(t)$ for an antiphase bimodal CT distribution $\left\{\phi_{j}(t\right.$ $\left.\left.+\tau_{k}\right)\right\}_{k=1, \ldots, l}$ have been introduced as alternatives to the stimulus locking indices $\lambda_{j}^{(1)}(t)$ and $\lambda_{j}^{(2)}(t)$ [Eqs. (18), (20), and (23)]. By the same token, the CT mean angular deviation $\Upsilon_{n, m}(t)$ for a unimodal CT distribution $\left\{\varphi_{n, m}\left(t+\tau_{k}\right)\right\}_{k=1, \ldots, l}$ is an alternative to the $n: m$ synchronization index $\sigma_{n, m}(t)$ [Eqs. (19) and (24)]. Applied to the signals under consideration, $\Lambda_{j}^{(\nu)}(t)$ and $\lambda_{j}^{(\nu)}(t) \quad(\nu=1,2)$ as well as $\Upsilon_{n, m}(t)$ and $\sigma_{n, m}(t)$ provide practically the same information, respectively.

In this context it should be mentioned that $\lambda_{j}^{(2)}(t)$ and $\Lambda_{j}^{(2)}(t)$ share a relevant drawback. Both are not specifically detecting an antiphase CT clustering. Not only an antiphase CT clustering [Figs. 5(1) and 5(p)], but also a simple reset [Figs. 5(c) and 5(g)] is related to significantly large values of $\lambda_{j}^{(2)}(t)$ and, correspondingly, to significantly small values of $\Lambda_{j}^{(2)}(t)$. For this reason, the antiphase CT clustering index $\alpha_{j}(t)$ from Eq. (21) has been introduced [29]. $\alpha_{j}(t)$ specifically detects two symmetric antiphase peaks of the distribution $\left\{\phi_{j}\left(t+\tau_{k}\right)\right\}_{k=1, \ldots, l}$ at time $t$. Only an antiphase CT clustering [Fig. 5(m)] - but not a simple reset [Fig. 5(d)]—is connected with significantly large values of $\alpha_{j}(t)$. With a decrease in symmetry of the antiphase CT clustering $\alpha_{j}(t)$ decreases [see Fig. 7 in Ref. [29] and Sec. III].

In circular statistics formula for skewness and kurtosis, i.e., for the third and the fourth moment, of a circular distribution have been derived $[33,57]$. To introduce mean phases $\Delta_{j}^{(\nu)}$ belonging to the stimulus locking indices from Eq. (18), I rewrite this formula and obtain

$$
\lambda_{j}^{(\nu)}(t) \exp \left[i \Delta_{j}^{(\nu)}(t)\right]=\frac{1}{l} \sum_{k=1}^{l} \exp \left[i \nu 2 \pi \phi_{j}\left(\tau_{k}+t\right)\right] .
$$

With this, the time-dependent skewness $s_{j}(t)$ of the CT distribution of the normalized phase of oscillator $j,\left\{\phi_{j}(t\right.$ $\left.\left.+\tau_{k}\right)\right\}_{k=1, \ldots, l}$ from Eq. (17), reads

$$
s_{j}(t)=\lambda_{j}^{(2)}(t) \sin \left[\Delta_{j}^{(2)}(t)-2 \Delta_{j}^{(1)}(t)\right],
$$

Ref. [33]. $s_{j}(t)$ vanishes if the CT distribution $\left\{\phi_{j}(t\right.$ $\left.\left.+\tau_{k}\right)\right\}_{k=1, \ldots, l}$ is symmetric around its peak at time $t$.

The time-dependent kurtosis $k_{j}(t)$ of the CT distribution of the normalized phase of oscillator $j,\left\{\phi_{j}\left(t+\tau_{k}\right)\right\}_{k=1, \ldots, l}$, is given by

$$
k_{j}(t)=\lambda_{j}^{(2)}(t) \cos \left[\Delta_{j}^{(2)}(t)-2 \Delta_{j}^{(1)}(t)\right],
$$

Ref. [33]. The kurtosis assesses to what extent a distribution is relatively flat or sharply peaked. One obtains an alternative measure for the kurtosis by the replacement $k_{j}(t)$ $\rightarrow k_{j}(t) /\left[\Lambda_{j}^{(1)}(t)\right]^{3}$, where the denominator serves to eliminate possible effects caused by dispersion [57]. $\Lambda_{j}^{(1)}(t)$ is the CT mean angular deviation for a unimodal CT distribution defined by Eq. (23). In a similar way, skewness and kurtosis can be determined for the CT distribution of the $n: m$ phase difference, $\left\{\varphi_{n, m}\left(t+\tau_{k}\right)\right\}_{k=1, \ldots, l}$ from Eq. (17).

It is important to note that skewness and kurtosis are meaningful only for unimodal distributions [33]. The present study is not focussing on how a stimulus leads to transient changes of particular features of the shape of unimodal distributions. Rather, more drastic effect such as transitions between uniform, unimodal, and multimodal distributions were studied. For this reason I did not determine skewness and kurtosis. However, in other numerical or experimental applications one might profit from these additional measures.

Previously, transient stimulus-locked phase dynamics and synchronization have been investigated in two-phase oscillators that were both stimulated, either simultaneously [28,29] or at different times (with a delay that did not exceed the time scale of the transients) [30]. Let us compare the results of these previous studies with the transmission dynamics found in the present study.

Hallmark of transmission. The maximal response of oscillator 2 in terms of a reset or a CT response clustering comes after both the maximal response of oscillator 1 and the transient stimulus-locked desynchronization/synchronization between the two oscillators, at least for values of the coupling strength $K$ that are reasonable from a biological standpoint, e.g., for $K$ up to, say, 5 [Figs. 2 and 3]. In contrast, if both oscillators are stimulated at the same time, the resets of both oscillators as well as the transient desynchronization/ synchronization occur at the same time. 
The delayed occurrence of the reset or the CT response clustering of the not directly stimulated oscillator is an important criterion for identifying how the action of a stimulus is transmitted within a network of neuronal oscillators. For instance, in a recent MEG study this criterion made it possible to identify which visual brain areas receive direct input from the retina and which get the input by transmission via the directly stimulated brain areas [31].

Stochastic resonance. If both oscillators are stimulated either at the same or at different times [30], the CT response clustering may come after a reset. In detuned oscillators this type of CT response clustering requires the presence of noise. In fact, by varying the noise amplitude, the extent of CT response clustering displays a stochastic resonance, i.e., the response clustering is strongest for an optimal, intermediate noise amplitude [28-30]. In contrast, the dynamical phenomena presented in this paper are not subject to a stochastic resonance. Rather they occur for vanishing noise, too (simulations not shown due to space constraints).

Fourier mode based indices vs Shannon entropy based indices. In case both oscillators were stimulated, the indices based on the Shannon entropy from Eqs. (25) and (26) were not sensitive enough to detect CT response clustering, whereas a reset always leads to an increase of these indices beyond the prestimulus level (i.e., beyond the 99th percentile of the corresponding prestimulus CT distribution) [28-30]. In contrast, in the present study it is different: Not only a reset (Fig. 2), but also a CT two clustering (Fig. 5) as well as a CT three clustering (Fig. 8) lead to an increase of the corresponding index beyond its prestimulus level.

In principle, the advantage of the Shannon entropy based indices would be that different types of responses, such as a simple reset or different sorts of CT response clustering, could be detected with only one index for each phase $\left[\mu_{j}\right.$ from Eq. (25)] and one index for the phase difference $\left[\eta_{n, m}\right.$ from Eq. (26)]. In this sense one could use the Shannon entropy based indices as screening indices. If a response of whatever kind would be detected with the Shannon entropy based indices, the Fourier mode based indices from Eqs. (18)-(22) could be used to specifically identify the type of response (reset or the particular sort of CT response clustering). However, since in case of two stimulated oscillators the Shannon entropy based indices fail in detecting events, and since in an experimental application it need not a priori be clear whether there is a transmission or not, the Shannon entropy based indices should not be used as screening indices.

The CT Kuiper test as screening test. Unlike the Shannon entropy based indices, both the CT Kuiper test for randomness of the CT distribution of the phase $\phi_{j}$ from Eq. (39) and the CT Kuiper test for randomness of the CT distribution of the $n: m$ phase difference $\varphi_{n, m}$ from Eq. (40) provide robust screening tests for stimulus locked dynamics of the phase and the $n: m$ phase difference. For the numerical signals under consideration, the CT Kuiper test turned out to be sensitive enough to detect all sorts of resetting, clustering, and synchronization phenomena considered in this study [Secs. V B, VII E, and $\mathrm{X}]$. Whenever the poststimulus $P$ values from Eqs. (39) and (40) leave the prestimulus baseline range (from the first to the 99th prestimulus percentile), one can use the resetting index $\rho_{j}$ from Eq. (20), the CT response clustering indices $\alpha_{j}$ and $\beta_{j}$ from Eqs. (21) and (22), and the $n: m$ synchronization index $\sigma_{n, m}$ from Eq. (19) to determine the concrete type of resetting, response clustering, synchronization, or desynchronization, respectively. In analogy to Eqs. (21) and (22), in case of $\nu$ equally spaced peaks in the CT distribution of, e.g., the phase $\phi_{j}$, and $\nu>3$, one can use the time-dependent CT $\nu$-clustering index of the $j$ th oscillator defined by $\lambda_{j}^{(\nu)}(t)-\lambda_{j}^{(1)}(t) \quad(\nu=4,5, \ldots) . \quad-1 \leqslant \lambda_{j}^{(\nu)}(t)$ $-\lambda_{j}^{(1)}(t) \leqslant 1$ is fulfilled for all times $t$, where $\nu$ Dirac-like symmetric and equally spaced peaks are connected with $\lambda_{j}^{(\nu)}(t)-\lambda_{j}^{(1)}(t)=1$.

According to the tests performed on synthetic responses in Sec. X, the CT Kolmogorov-Smirnov test from Eqs. (35) and (36), the CTCC from Eq. (42) and the CTSCC from Eq. (43) are no appropriate measures for estimating stimuluslocked dynamics, since they produce artificial oscillations that are not related to the stimulus-locked dynamics under study. When applying the entropy based stimulus locking index $\mu_{j}$ from Eq. (25) as well as the entropy based 1:1 synchronization index $\eta_{1,1}$ from Eq. (26), one has to keep in mind that in case of sharp CT distributions binning-induced artificial oscillations occur [Figs. 11(g) and 11(h)]. In particular, in applications to noisy experimental signals, the CT distributions are typically not sharp, so that the binning may, hence, cause no problems.

The same artificial oscillations as demonstrated in Sec. X occur in case of an $n: m$ phase relationship. In other words, also for $n: m$ synchronization the CT Kolmogorov-Smirnov test, the CTCC, and the CTSCC produce similar artificial oscillations that are not related to the stimulus-locked dynamics (not shown because of space constraints). Also, the entropy based $n: m$ synchronization index $\eta_{n, m}$ produces binning-induced oscillations in case of sharp CT distributions of the $n: m$ phase difference.

Similar to the resetting index $\rho_{j}$ from Eq. (20) and the $n: m$ synchronization index $\sigma_{n, m}$ from Eq. (19) [Figs. 11(d)-11(f)], also the mean angular deviations from Eqs. (23) and (24), the antiphase CT clustering index from Eq. (21), and the CT three-clustering index from Eq. (22) cause no artificial oscillations. This follows directly from their definitions.

For the study of phase resetting in EEG signals in sensory stimulation experiments a quantity has been used which is comparable to the resetting index $\rho_{j}$ from Eq. (20), and which was based on a wavelet transformation and denoted as "phase-locking factor" $[9,58]$. The results presented here as well as previous findings [28-30] clearly show that one cannot use only the resetting index $\rho_{j}$ from Eq. (20) or only the antiphase CT clustering index $\alpha_{j}$ from Eq. (21) as sort of a screening index. Rather, the CT Kuiper test (Sec. III E) serves as screening test, at least for the signals analyzed in this study. As explained above, the CT Kuiper test detects stimulus-locked epochs, whereas the specific nature of such an epoch (reset, CT response clustering, synchronization, desynchronization) can be detected with the indices from Sec. III B. Up to now, only reset and antiphase CT response clustering have been found in experimental data [31]. As an alternative, instead of first applying the CT Kuiper test, one 
could start with a visual inspection of the $\mathrm{CT}$ distributions from Eq. (17) and then decide which Fourier based index is most appropriate. However, such an approach is not feasible if thousands of signals, e.g., thousands of currents in different brain volume elements have to be analyzed (see Ref. [31]).

CT averaging. Antiphase CT response clustering cannot be detected with CT averaging from Eq. (1), especially, if it is close to symmetrical, i.e., if the two antiphase responses appear with similar frequency across trials. This holds, in general, and was thus observed not only in the present study, but also in case of two stimulated oscillators [28-30]. In other words, more complex types of responses than a simple reset escape detection with the $\mathrm{CT}$ averaging, the standard analysis tool in medicine and biology.

CT standard deviation. In case both oscillators are stimulated [28-30] as well as only one oscillator is stimulated [Figs. 7(b) and 7(d)], the CT standard deviation displays an artificial oscillation with typically twice the frequency of the analyzed oscillator [Figs. 11(s) and 11(t)]. Accordingly, the CT standard deviation is not appropriate for the analysis of stimulus-locked responses.

CT cross correlation. After a reset of both oscillators the CT cross correlation from Eq. (42) displays artificial oscillations [28-30] [Fig. 11(r)]. They arise because the CT cross correlation depends not only on the phase difference, but also on the phase of each individual oscillator. Here I have demonstrated that the CT cross correlation need not display such artificial oscillations, provided only one oscillator is reset. However, also in this case the CT cross correlation is not able to detect relevant transient phenomena, such as the pronounced stimulus-locked transient desynchronization shown in Figs. 5(i)-5(k) [cf. Fig. 7(e)]. Thus, CT cross correlation is not a reliable method for the analysis of transient stimuluslocked dynamics. Also, the CT sign cross correlation from Eq. (43) produces the same artifacts as the CT cross correlation [Fig. 11(u)] and is, hence, not reliable, too.

The data analysis from Sec. III can directly be applied to experimental data. In case of discrete signals such as timing sequences of spiking neurons the phase can, e.g., be estimated with linear interpolation. In case of continuous experi- mental data, also the amplitudes of the oscillators have to be studied. For this, an oscillatory signal $x_{j}(t)$, e.g., a particular brain rhythm, is extracted out of a measured signal by means of bandpass filtering. The Hilbert transform $x_{j}^{\mathrm{H}}(t)$ of $x_{j}(t)$ yields instantaneous phase $\psi_{j}(t)$ and instantaneous amplitude $A_{j}(t)$ of $x_{j}(t)$ according to $x_{j}(t)+x_{j}^{\mathrm{H}}(t)=A_{j}(t) \exp \left[i \psi_{j}(t)\right]$ [59]. The Hilbert transform is generated by a filter, which causes a phase shift of $\pi / 2$ for all frequencies. Alternatively, also a wavelet approach can be used for the phase determination [60]. The amplitudes $A_{j}$ of the oscillators can, in principle, be averaged across trials as done in Eq. (1) with the signals. With such an analysis, however, qualitatively different transients of the amplitudes cannot be detected. It is superior to check for CT clustering of amplitude responses by use of the stochastic phase resetting analysis from Sec. III. To this end, similar to Eq. (17) I introduce CT distributions of the amplitudes with $\left\{A_{j}\left(t+\tau_{k}\right)\right\}_{k=1, \ldots, l}$ and evaluate them in a comparable way as defined by Eqs. (18) and (19) for the phases. Obviously, for the phase oscillator model under consideration there was no need for an amplitude analysis, since the amplitude of a phase oscillator is constant.

One motivation behind the present study is to reveal basic features of stimulus-locked transient dynamics of twocoupled oscillators. The other motivation is to contribute to a more solid basis for analyzing stimulus-locked responses of interacting brain rhythms as measured, e.g., with EEG and MEG. Actually, the CT stochastic phase resetting analysis from Sec. III has allowed to show that brain areas may react to simple visual stimuli in qualitatively different ways across trials [31]. This finding illustrates the limitations of the standard CT analysis techniques (Sec. IV) used in medicine and neuroscience.

\section{ACKNOWLEDGMENTS}

This work was supported by the Volkswagen Foundation (Grant No. 76761), the German Israeli Foundation (Grant No. I-667-81.1/2000), and the German Academic Exchange Service (DAAD, Grant No. D/0247269). I am grateful to Michael Schiek for fruitful discussions.
[1] H. Haken, Advanced Synergetics (Springer, Berlin, 1983).

[2] Y. Kuramoto, Chemical Oscillations, Waves, and Turbulence (Springer, Berlin, 1984).

[3] A. T. Winfree, The Geometry of Biological Time (Springer, Berlin, 1980).

[4] A. B. Neiman and D. F. Russell, Phys. Rev. Lett. 88, 138103 (2002); B. Naundorf and J. A. Freund, Phys. Rev. E 66, 040901(R) (2002); B. Lindner et al., ibid. 66, 031916 (2002); S. Bahar et al., ibid. 65, 050901 (2002); C. R. Laing and A. Longtin, ibid. 67, 051928 (2003).

[5] P. A. Tass, Phase Resetting in Medicine and Biology. Stochastic Modelling and Data Analysis (Springer, Berlin, 1999).

[6] H. Steriade et al., Thalamic Oscillations and Signaling (Wiley, New York, 1990).
[7] K. H. Chiappa, Evoked Potentials in Clinical Medicine (Raven Press, New York, 1983).

[8] B. Sayers et al., Nature (London) 247, 481 (1974).

[9] S. Makeig et al., Science 295, 690 (2002).

[10] G. D. Dawson, Electroencephalogr. Clin. Neurophysiol. 6, 153 (1954).

[11] M. Hämäläinen et al., Rev. Mod. Phys. 65, 413 (1993).

[12] A. Frank et al., Physica D 144, 62 (2000).

[13] J. A. S. Kelso et al., Phys. Lett. A 169, 134 (1992).

[14] A. Daffertshofer et al., Phys. Lett. A 266, 290 (2000).

[15] A. Fuchs et al., Phys. Lett. A 266, 303 (2000).

[16] H. J. Heinze and H. Kunkel, Methods Inf. Med. 23, 29 (1984).

[17] D. O. Walter, Electroencephalogr Clin. Neurophysiol. Suppl. 27, 61 (1969). 
[18] D. J. Doyle, Electroencephalogr. Clin. Neurophysiol. 38, 533 (1975).

[19] J. P. De Weerd, Biol. Cybern. 41, 211 (1981).

[20] J. P. De Weerd and J. I. Kap, Biol. Cybern. 41, 223 (1981).

[21] R. R. Coifman and M. Y. Wickerhauser, Electroencephalogr Clin. Neurophysiol. Suppl. 45, 57 (1996).

[22] S. J. Schiff et al., Electroencephalogr. Clin. Neurophysiol. 91, 442 (1994).

[23] S. J. Schiff et al., Opt. Eng. 33, 2162 (1994).

[24] V. J. Samar et al., Brain Cogn 27, 398 (1995).

[25] A. Effern et al., Physica D 140, 257 (2000).

[26] R. Hari and R. Salmelin, Trends Neurosci. 20, 44 (1997).

[27] Handbook of Biological Physics, edited by C. Gielen and F. Moss (Elsevier, Amsterdam, 2001), Vol. IV

[28] P. A. Tass, Europhys. Lett. 59, 199 (2002).

[29] P. A. Tass, Chaos 13, 364 (2003).

[30] P. A. Tass, Phys. Rev. E 67, 051902 (2003).

[31] P. A. Tass et al. (unpublished).

[32] C. W. Gardiner, Handbook for Stochastic Methods for Physics, Chemistry and the Natural Sciences, 2nd ed. (Springer, Berlin, 1985).

[33] E. Batschelet, Circular Statistics in Biology (Academic Press, London, 1981).

[34] E. Batschelet, Statistical Methods for the Analysis of Problems in Animal Orientation and Certain Biological Rhythms (Amer. Inst. Biol. Sci., Washington, D.C., 1965).

[35] R. K. Otnes and L. Enochson, Digital Time Series Analysis (Wiley, New York, 1972).

[36] J. L. Hodges, Jr., Ann. Math. Stat. 26, 523 (1955).

[37] B. Ajne, Biometrika 55, 343 (1968).

[38] G. K. Bhattacharyya and R. A. Johnson, Biometrika 56, 446 (1969).
[39] J. S. Rao, Indian J. Stat., Ser. B 38, 329 (1976).

[40] N. F. Laubscher and G. J. Rudolph, Ann. Math. Stat. 38, 964 (1967).

[41] J. S. Rao, Z. Wahrscheinlichkeitstheor. Verwandte Geb. 22, 33 (1972).

[42] G. S. Watson, Biometrika 48, 109 (1961).

[43] N. H. Kuiper, Proc. K. Ned. Akad. Wet., Ser. A: Math. Sci. 63, 38 (1960)

[44] M. A. Stephens, Biometrika 52, 309 (1965).

[45] I. G. Abrahamson, Ann. Math. Stat. 38, 1475 (1967).

[46] U. R. Maag, Ann. Stat. 1, 1185 (1973).

[47] K. Bucher and P. Bättig, Helv. Physiol. Pharmacol. Acta 18, 25 (1960).

[48] P. Engel et al., Pfluegers Arch. Gesamte Physiol. Menschen Tiere 298, 258 (1967).

[49] M. Schiek, Ph.D. thesis, University of Aachen, 1998 (unpublished).

[50] W. J. P. Barnes, J. Comput. Phys. 96, 237 (1975).

[51] W. H. Press et al., Numerical Recipes in C, 2nd ed. (Cambridge University Press, Cambridge, 1992).

[52] H. Forssberg et al., Brain Res. 85, 103 (1975).

[53] P. A. Tass et al., Phys. Rev. Lett. 81, 3291 (1998).

[54] P. A. Tass et al., Phys. Rev. Lett. 90, 088101 (2003).

[55] R. L. Stratonovich, Topics in the Theory of Random Noise (Gordon and Breach, New York, 1963).

[56] M. G. Rosenblum et al., Phys. Rev. Lett. 76, 1804 (1996).

[57] K. V. Mardia, Statistics of Directional Data (Academic Press, London, 1972).

[58] C. Tallon-Baudry et al., J. Neurosci. 16, 4240 (1996).

[59] P. Panter, Modulation, Noise, and Spectral Analysis (McGrawHill, New York, 1965).

[60] R. Quian Quiroga et al., Phys. Rev. E 65, 041903 (2002). 